\title{
Matching effective chiral Lagrangians with dimensional and lattice regularizations
}

\author{
F. Niedermayer ${ }^{a}$ and P. Weisz ${ }^{b}$ \\ ${ }^{a}$ Albert Einstein Center for Fundamental Physics, Institute for Theoretical Physics, \\ University of Bern, \\ Bern, 3012, Switzerland \\ ${ }^{b}$ Max-Planck-Institut für Physik, \\ Munich, 80805, Germany \\ E-mail: niedermayer@itp.unibe.ch, pew@mpp.mpg.de
}

ABSTRACT: We compute the free energy in the presence of a chemical potential coupled to a conserved charge in effective $\mathrm{O}(n)$ scalar field theory (without explicit symmetry breaking terms) to third order for asymmetric volumes in general $d$-dimensions, using dimensional (DR) and lattice regularizations. This yields relations between the 4-derivative couplings appearing in the effective actions for the two regularizations, which in turn allows us to translate results, e.g. the mass gap in a finite periodic box in $d=3+1$ dimensions, from one regularization to the other. Consistency is found with a new direct computation of the mass gap using DR. For the case $n=4, d=4$ the model is the low-energy effective theory of QCD with $N_{\mathrm{f}}=2$ massless quarks. The results can thus be used to obtain estimates of low energy constants in the effective chiral Lagrangian from measurements of the low energy observables, including the low lying spectrum of $N_{\mathrm{f}}=2$ QCD in the $\delta$-regime using lattice simulations, as proposed by Peter Hasenfratz, or from the susceptibility corresponding to the chemical potential used.

KEywords: Effective field theories, Sigma Models, Lattice Quantum Field Theory

ArXiv EPrint: 1601.00614v2

We dedicate this paper to the memory of Peter Hasenfratz, a great physicist, our good friend and colleague. 


\section{Contents}

1 Introduction 1

2 The free energy on the lattice 3

2.1 The effective lattice action 3

2.2 Perturbative expansion 5

2.3 The chemical potential $\quad 7$

2.3.1 Computation of $F_{1}$ up to $\mathrm{O}\left(g_{0}^{2}\right) \quad 9$

2.3.2 Computation of $F_{2}$ up to $\mathrm{O}\left(g_{0}^{2}\right) \quad 9$

$\begin{array}{lll}2.3 .3 & \text { Summary } & 11\end{array}$

$\begin{array}{lll}2.4 & \text { Renormalization on the lattice } & 11\end{array}$

2.4.1 Case $d=2 \quad 12$

2.4.2 Case $d=3 \quad 13$

2.4.3 Case $d=4 \quad 14$

3 The free energy with dimensional regularization $\quad 17$

$\begin{array}{ll}3.1 \text { The effective action } & 17\end{array}$

$\begin{array}{lll}3.2 & \text { Perturbative expansion } & 18\end{array}$

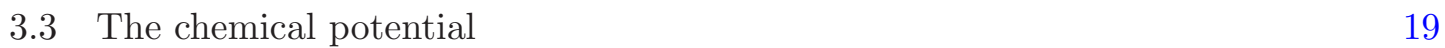

$\begin{array}{lll}3.4 & \text { Summary } & 22\end{array}$

$\begin{array}{ll}3.5 & \text { Case } n=2\end{array} 22$

$\begin{array}{lll}3.6 & \text { Case } d=2 & 23\end{array}$

$\begin{array}{lll}3.7 & \text { Case } d=3 & 24\end{array}$

3.8 Case $d=4 \quad 24$

4 Matching the effective actions for $d=2$ and $d=4 \quad 26$

$\begin{array}{lll}4.1 & \text { Case } d=2 & 26\end{array}$

4.2 Case $d=4 \quad 28$

5 The mass gap $\quad 30$

6 Conclusions $\quad 32$

A The terms $B_{4}^{(i)}$ and $C_{4}^{(i)} \quad 35$

$\begin{array}{ll}\text { B Some lattice momentum sums } & 36\end{array}$

$\begin{array}{ll}\text { C Correlators appearing in } \boldsymbol{F}_{\boldsymbol{r}} \text { with lattice regularization } & \mathbf{3 7}\end{array}$

$\begin{array}{lll}\text { C.1 Correlators appearing in } F_{1,0} \text { and } F_{1,1} & 37\end{array}$

C.2 Correlators appearing in $F_{2,0}$ and $F_{2,1} \quad 38$

$\begin{array}{lll}\text { C.3 Computation of } F_{3} \text { up to } \mathrm{O}\left(g_{0}^{2}\right) & 39\end{array}$

$\begin{array}{ll}\text { C.3.1 Computation of } F_{3,1}^{(i)} & 40\end{array}$ 
C.4 Computation of $F_{4}$ up to $\mathrm{O}\left(g_{0}^{2}\right)$

C.4.1 Computation of $F_{4,1}^{(i)} \quad 42$

C.5 Computation of $F_{5}$ up to $\mathrm{O}\left(g_{0}^{2}\right)$

D The $n=2$ case with lattice regularization 43

\section{Introduction}

There is now much confidence that Quantum Chromodynamics (QCD) is the theory of the strong interactions, and there is good evidence that in this theory with massless quarks chiral flavor symmetry is spontaneously broken. The low energy phenomena in systems with spontaneously broken symmetry are governed by the dynamics of the Goldstone bosons (pions in the case of QCD). This can be described by an effective field theory, and the calculations can be performed by chiral perturbation theory $\chi \mathrm{PT}[1,2]$.

The interplay between $\chi \mathrm{PT}$ and QCD has been extremely fruitful. In early times of lattice simulations of QCD when light dynamical quarks could not be simulated efficiently, $\chi \mathrm{PT}$ was used to extrapolate the data to smaller pion masses $m_{\pi}$. Lately, since simulations at physical pion masses became feasible, one can use lattice data to obtain the parameters in the chiral Lagrangian, the pion decay constant $F_{\pi}$ and the low energy constants (LEC's), from the underlying microscopic theory QCD more precisely than from phenomenology. For a detailed summary of various determinations of the LEC's the reader is referred to the FLAG review [3].

Both $\chi \mathrm{PT}$ computations and lattice simulations of QCD can be done in special environments where physical experiments cannot be envisaged. One can study the dependence on parameters (such as quark masses), and one can place the system into a space-time box of size $L_{t} \times L_{s}^{d-1}$ and study the dependence of physical quantities on the box size $L_{s}$ of the order a few fermi. Leutwyler was the first to systematize the different regimes of QCD in a finite box [4]. One special environment is the so called $\delta$-regime where the system is in a periodic spatial box of sides $L_{s}$ and $m_{\pi} L_{s}$ is small (i.e. small or zero quark mass) whereas $F_{\pi} L_{s}$ is large.

In 2009 Hasenfratz [5] pointed out that promising observables in the $\delta$-regime are the low lying stable masses. Firstly measuring low lying stable masses to good precision is among the easiest numerical tasks. Secondly the finite box size introduces an infra-red cutoff which allows to study the chiral limit in a first stage and switching on the symmetry breaking terms later.

For massless two-flavor QCD the relevant $\chi \mathrm{PT}$ has $\mathrm{SU}(2) \times \mathrm{SU}(2) \simeq O(4)$ symmetry. It has been shown by Leutwyler [4] that in the leading order of $\chi \mathrm{PT}$, with general (unbroken) $\mathrm{O}(n)$ symmetry (and $d=4$ ), the spectrum is given by a quantum mechanical rotator $E(l)=l(l+n-2) /(2 \Theta), l=0,1,2, \ldots$, the "angular momentum" being the $\mathrm{O}(n)$ isospin, with moment of inertia $\Theta=F^{2} L_{s}^{3}$ fixed by the decay constant $F$ (in the chiral limit). 
The next-to-leading order (NLO) term of the expansion in $1 /\left(F^{2} L_{s}^{2}\right)$ has been calculated in [6]. The level spectrum is to this order still governed solely by $F$, so an evaluation of this spectrum on the lattice potentially gives a good initial estimate of $F$. Since the NLO correction turned out to be large, however, it was important to calculate the nextto-next-to-leading order (NNLO) term; furthermore the chiral logs and the LEC's $l_{1}, l_{2}$ (in the 4-derivative terms in the effective action) first appear at this order.

Two independent results for the NNLO correction have been presented. The first is by Hasenfratz [5] using dimensional regularization (DR). His procedure, which was quite involved, was to consider a volume infinite in the time direction, and to separate the degrees of freedom in the $\delta$-regime into (spatially constant) slow and fast modes. The latter are then integrated out (treated in PT) resulting in an effective Lagrangian for the slow modes, an $\mathrm{O}(n)$ rotator with a modified moment of inertia, whose energy excitations are much smaller than those of the standard Goldstone boson excitations carrying finite momenta $\geq 2 \pi / L_{s}$.

The second computation was by Niedermayer and Weiermann [7] using lattice regularization; it involved generalizing the computation of the small-volume mass gap in the $2 \mathrm{~d}$ $\mathrm{O}(n)$ non-linear sigma-model by Lüscher, Weisz, and Wolff [8] to higher dimensions $d>2$.

Of course the physical content of a QFT is independent of regularization. The matching of UV regularizations of renormalizable asymptotically free theories can be obtained by determining the ratio of $\Lambda$-parameters which just involves a 1-loop calculation. Here we have an effective QFT and the matching of different regularizations in such theories is, as far as we know, still a relatively untouched problem. In particular the results of the two NNLO computations referred to above could not be quantitatively compared, apart from the chiral logs, since relations between the couplings of the 4-derivative terms in the effective Lagrangians were unknown. In this paper we have closed this gap.

Here we compute the change in the free energy due to a chemical potential coupled to a conserved charge in the non-linear $\mathrm{O}(n)$ sigma model with two regularizations, lattice regularization (with standard action) in section 2 and DR in section 3. The computation is performed in a general $d$-dimensional volume with periodic boundary conditions in all directions. The volume is left asymmetric. This freedom allows us for $d=4$ in section 4 to establish two independent relations among the 4-derivative couplings appearing in the effective actions of the two regularizations. These relations in turn allow us to convert results for physical quantities computed by the lattice regularization to those involving scales introduced in DR. Computations on the lattice, although algebraically more involved than analogous continuum computations, have the advantage that they are conceptually "fool-proof". Computations with DR are however often tricky starting at two loops.

In particular one of the relations referred to above allow us to convert the result of the mass gap computed on the lattice in [7] to DR (in section 5). Unfortunately the outcome of this does not agree with the result of Hasenfratz [5]. We thus recomputed the mass gap with DR and thereby obtained a result in complete agreement with that translated from the lattice. We are thus quite confident that it is correct.

The sums and integrals which appear in our computation, in particular the two-loop massless sunset diagram, are treated in a separate accompanying paper [9]. 
In this paper we do not consider explicit $\mathrm{O}(n)$ symmetry breaking. In QCD the effect of including a small explicit symmetry breaking (a small quark mass) has been done to LO in [4], and to NLO by Weingart [10, 11]. In a recent paper Matzelle and Tiburzi [12] study the effect of small symmetry breaking in the QM rotator picture, and extend the results for small non-zero temperatures.

The proposal to use the mass gap in the delta regime to determine the LEC's of QCD has its own advantages and disadvantages. As opposed to a similar problem in the $\epsilon$-regime one does not need the value of the (finite-volume, renormalized) condensate, which is a nontrivial task. It is rather insensitive to the LEC's $l_{1}$ and $l_{2}$, which also means that it is a convenient quantity to determine $F$. A computational difficulty is that one needs a largely elongated lattice in the time direction. To follow the decay of the correlation function one needs $m\left(L_{s}\right) L_{t} \gg 1$ which means $L_{t} / L_{s} \gg F^{2} L_{s}^{2}>1$. (The last condition should hold since we have an expansion in $1 /\left(F^{2} L_{s}^{2}\right)$ ). Another problem - which is, however, not specific for the $\delta$-regime - is to use a chirally invariant fermion action satisfying the Ginsparg-Wilson relation, if one would like to avoid the extra tuning of the bare quark mass approaching the chiral limit.

There are other important physical systems where the order parameter of the spontaneous symmetry breaking is an $\mathrm{O}(n)$ vector. In particular in condensed matter physics, anti-ferromagnetic layers are described by $\mathrm{O}(3)$ for $d=3$. Here the NNLO computation is complete to order $1 / L_{s}^{2}$; for details and comparisons to experiment see ref. [6].

\section{The free energy on the lattice}

In this section we work with a hyper-cubic $d$-dimensional lattice of volume $V=L_{t} \times$ $L_{s}^{d_{s}}, d_{s}=d-1$. Define the aspect ratio $\ell \equiv L_{t} / L_{s}$.

The dynamical variables are spins $S_{a}(x), a=1, \ldots, n$ of unit length $\mathbf{S}(x)^{2}=1$ with periodic boundary conditions in all directions $\mathbf{S}\left(x+L_{t} \hat{0}\right)=\mathbf{S}(x)=\mathbf{S}\left(x+L_{s} \hat{k}\right), k=$ $1, \ldots, d_{s}$, where $\hat{\mu}$ is the unit vector in the $\mu$-direction. We will often set the lattice spacing $a$ to 1 and will restore it again only in selected equations.

\subsection{The effective lattice action}

The effective lattice action $\mathcal{A}$ is a sum over terms

$$
\mathcal{A}=A_{2}+A_{4}+\ldots
$$

where the classical continuum limit of $A_{2 r}$ has $2 r$ derivatives. In this paper we will only consider the expansion up to and including four derivatives.

For $A_{2}$ we take the standard lattice action:

$$
A_{2}=\frac{1}{2 g_{0}^{2}} \sum_{x \mu} \partial_{\mu} \mathbf{S}(x) \cdot \partial_{\mu} \mathbf{S}(x)=-\frac{1}{g_{0}^{2}} \sum_{x \mu} \mathbf{S}(x) \cdot \mathbf{S}(x+\hat{\mu})+\text { const },
$$


where $g_{0}$ is the bare coupling and $\partial_{\mu}$ is the lattice difference operator (we will also need the backward difference operator $\partial_{\mu}^{*}$ )

$$
\begin{aligned}
& \partial_{\mu} f(x)=f(x+\hat{\mu})-f(x), \\
& \partial_{\mu}^{*} f(x)=f(x)-f(x-\hat{\mu}) .
\end{aligned}
$$

The most general form of the four derivative terms is given by $[6]$

$$
A_{4}=\sum_{i=1}^{5} \frac{g_{4}^{(i)}}{4}\left[A_{4}^{(i)}-c^{(i)} \sum_{x \mu} \partial_{\mu} \mathbf{S}(x) \cdot \partial_{\mu} \mathbf{S}(x)\right],
$$

where

$$
\begin{aligned}
& A_{4}^{(1)}=\sum_{x} \square \mathbf{S}(x) \cdot \square \mathbf{S}(x), \\
& A_{4}^{(2)}=\sum_{x \mu \nu}\left[\partial_{\mu} \mathbf{S}(x) \cdot \partial_{\mu} \mathbf{S}(x)\right]\left[\partial_{\nu} \mathbf{S}(x) \cdot \partial_{\nu} \mathbf{S}(x)\right], \\
& A_{4}^{(3)}=\sum_{x \mu \nu}\left[\partial_{\mu} \mathbf{S}(x) \cdot \partial_{\nu} \mathbf{S}(x)\right]\left[\partial_{\mu} \mathbf{S}(x) \cdot \partial_{\nu} \mathbf{S}(x)\right], \\
& A_{4}^{(4)}=A_{4}^{(4 a)}-\frac{1}{d+2}\left(A_{4}^{(2)}+2 A_{4}^{(3)}\right), \\
& A_{4}^{(5)}=A_{4}^{(5 a)}-\frac{1}{d+2}\left(2 A_{4}^{(5 b)}+A_{4}^{(5 c)}\right),
\end{aligned}
$$

and

$$
\begin{aligned}
& A_{4}^{(4 a)}=\sum_{x \mu}\left(\partial_{\mu} \mathbf{S}_{x} \cdot \partial_{\mu} \mathbf{S}_{x}\right)^{2}, \\
& A_{4}^{(5 a)}=\sum_{x \mu} \square_{\mu} \mathbf{S}(x) \cdot \square_{\mu} \mathbf{S}(x), \\
& A_{4}^{(5 b)}=A_{4}^{(1)}, \\
& A_{4}^{(5 c)}=\sum_{x \mu \nu} \partial_{\mu} \partial_{\mu} \mathbf{S}(x) \cdot \partial_{\nu} \partial_{\nu} \mathbf{S}(x),
\end{aligned}
$$

where

$$
\square_{\mu} \equiv \partial_{\mu} \partial_{\mu}^{*}, \quad \square=\sum_{\mu} \square_{\mu}
$$

In (2.5) we subtract a term proportional to the leading action $A_{2}$ from each of the 4derivative interactions. The coefficients $c^{(i)}$ serve to remove the power-like divergence $1 / a^{p}$ from the contribution of the corresponding operator, (note $c^{(4)}=0$ ). The subtracted operators then renormalize multiplicatively.

The set of five operators above is redundant. ${ }^{1}$ One can use this observation to eliminate, say, the coupling $g_{4}^{(1)}$ (as in [6]), or alternatively, to check that the final result for physical quantities depends only on the sum of the couplings, $g_{4}^{(1)}+g_{4}^{(2)}$.

${ }^{1}$ As explained in [6], changing the field variable

$$
\mathbf{S}(x) \rightarrow[\mathbf{S}(x)+\alpha \square \mathbf{S}(x)] /|\mathbf{S}(x)+\alpha \square \mathbf{S}(x)|
$$


The total action including only terms to this order is given by

$$
\mathcal{A}=Z_{4} A_{2}+\sum_{i=1}^{5} \frac{g_{4}^{(i)}}{4} A_{4}^{(i)},
$$

where

$$
Z_{4} \equiv 1-\frac{1}{2} g_{0}^{2} \sum_{i=1}^{5} g_{4}^{(i)} c^{(i)}
$$

\subsection{Perturbative expansion}

After separating the zero mode as in [13] and changing to $\vec{\pi}$ variables (with $\sum_{x} \vec{\pi}(x)=0$ ) according to $\mathbf{S}=\left(g_{0} \vec{\pi}, \sqrt{1-g_{0}^{2} \vec{\pi}^{2}}\right)$ we have

$$
A_{2, \mathrm{eff}}[\vec{\pi}]=A_{2}[\vec{\pi}]+A_{2, \text { measure }}[\vec{\pi}]+A_{2, \text { zero }}[\vec{\pi}]
$$

with

$$
\begin{aligned}
A_{2, \text { measure }}[\vec{\pi}] & =\sum_{x} \ln \left[1-g_{0}^{2} \vec{\pi}(x)^{2}\right]^{\frac{1}{2}}, \\
A_{2, \text { zero }}[\vec{\pi}] & =-n_{1} \ln \frac{1}{V} \sum_{x}\left[1-g_{0}^{2} \vec{\pi}(x)^{2}\right]^{\frac{1}{2}},
\end{aligned}
$$

where

$$
n_{1} \equiv n-1
$$

$A_{2, \text { eff }}$ has a perturbative expansion

$$
A_{2, \mathrm{eff}}=A_{2,0}+g_{0}^{2} A_{2,1}+g_{0}^{4} A_{2,2}+\ldots
$$

where (here we will need only $A_{2,0}$ and $A_{2,1}$ ):

$$
\begin{aligned}
& A_{2,0}=\frac{1}{2} \sum_{x} \partial_{\mu} \vec{\pi}(x) \cdot \partial_{\mu} \vec{\pi}(x), \\
& A_{2,1}=A_{2,1}^{(a)}+A_{2,1}^{(b)}, \\
& A_{2,1}^{(a)}=-\frac{1}{2}\left(1-\frac{n_{1}}{V}\right) \sum_{x} \vec{\pi}(x)^{2}, \\
& A_{2,1}^{(b)}=\frac{1}{8} \sum_{x} \partial_{\mu}\left[\vec{\pi}(x)^{2}\right] \partial_{\mu}\left[\vec{\pi}(x)^{2}\right] .
\end{aligned}
$$

We expand the couplings of the 4-derivative terms according to

$$
g_{4}^{(i)}=\sum_{r=0} g_{4, r}^{(i)} g_{0}^{2 r}
$$

the leading term of the effective action produces 4-derivative terms:

$$
\frac{1}{2} \sum_{x \mu} \partial_{\mu} \mathbf{S}(x) \cdot \partial_{\mu} \mathbf{S}(x) \rightarrow \frac{1}{2} \sum_{x \mu} \partial_{\mu} \mathbf{S}(x) \cdot \partial_{\mu} \mathbf{S}(x)-\alpha\left(A_{4}^{(1)}-A_{4}^{(2)}\right)+\ldots
$$

up to terms with higher derivatives. 
Noting that the coefficients $c^{(i)}$ defined in (2.5) are of order $g_{0}^{2}$ :

$$
c^{(i)}=\bar{c}^{(i)} g_{0}^{2}+\ldots
$$

the renormalization constant $Z_{4}$ has a perturbative expansion

$$
Z_{4}=1+\sum_{r=2} Z_{4, r} g_{0}^{2 r}, \quad\left(Z_{4,1}=0\right)
$$

with

$$
Z_{4,2}=-\frac{1}{2} \sum_{i=1}^{5} g_{4,0}^{(i)} \bar{c}^{(i)} .
$$

The total effective action has a perturbative expansion of the form

$$
\mathcal{A}=\sum_{r=0} \mathcal{A}_{r} g_{0}^{2 r}
$$

with

$$
\mathcal{A}_{0}=A_{2,0},
$$

and

$$
\mathcal{A}_{1}=A_{2,1}+\sum_{i=1}^{5} \frac{g_{4,0}^{(i)}}{4} A_{4,1}^{(i)},
$$

with

$$
\begin{aligned}
& A_{4,1}^{(1)}=\sum_{x} \square \vec{\pi}(x) \cdot \square \vec{\pi}(x), \\
& A_{4,1}^{(i)}=0, \quad i=2,3,4, \\
& A_{4,1}^{(5)}=A_{4,1}^{(5 a)}-\frac{1}{d+2}\left(2 A_{4,1}^{(5 b)}+A_{4,1}^{(5 c)}\right),
\end{aligned}
$$

with

$$
\begin{aligned}
& A_{4,1}^{(5 a)}=\sum_{x \mu} \square_{\mu} \vec{\pi}(x) \cdot \square_{\mu} \vec{\pi}(x), \\
& A_{4,1}^{(5 b)}=A_{4,1}^{(1)}, \\
& A_{4,1}^{(5 c)}=\sum_{x \mu \nu} \partial_{\mu} \partial_{\mu} \vec{\pi}(x) \cdot \partial_{\nu} \partial_{\nu} \vec{\pi}(x) .
\end{aligned}
$$

The perturbative coefficients of expectation values are sums of expectation values of products of $\vec{\pi}$ fields with respect to the Gaussian free field action $\mathcal{A}_{0}$ which are denoted by $\langle\ldots\rangle_{0}$. In particular the 2-point function is given by

$$
\left\langle\pi_{a}(x) \pi_{b}(y)\right\rangle_{0}=\delta_{a b} G(x-y),
$$

with the free propagator

$$
G(x)=\frac{1}{V} \sum_{p}^{\prime} \frac{\mathrm{e}^{i p x}}{\hat{p}^{2}}, \quad \hat{p}_{\mu}=2 \sin \frac{p_{\mu}}{2},
$$

where the sum is over momenta $p_{0}=2 \pi n_{0} / L_{t}, n_{0}=0, \ldots, L_{t}-1$ and $p_{k}=2 \pi n_{k} / L_{s}, n_{k}=$ $0, \ldots, L_{s}-1$ and the prime on the sum means that $p=0$ is omitted. 


\subsection{The chemical potential}

The action considered in this paper has no explicit $\mathrm{O}(n)$ ("isospin") symmetry breaking term. In an infinite volume the symmetry is broken spontaneously, Goldstone bosons (massless pions for QCD) appear, and the ground state of the theory is a condensed state of the Goldstone bosons. However, spontaneous isospin symmetry breaking is precluded in the present finite volume setup.

Because of the symmetry the isospin charge and its component $Q_{12}$ are well defined, conserved quantities; their values characterize a given eigenstate of the Hamiltonian (or transfer matrix). These numbers do not depend on the details of the regularization and renormalization. One introduces the chemical potential by modifying the transfer matrix ${ }^{2}$ $\hat{T}(a) \rightarrow \hat{T}(a) \mathrm{e}^{a h Q_{12}}$. By this one only re-weights the states in the partition function, shifting their energy by $h Q_{12}$, hence the $h$-dependent part of the free energy should not depend on these details either. Hence by comparing this quantity in two different regularizations one can relate the corresponding couplings.

In terms of the action introducing the chemical potential is done by the replacement

$$
\mathbf{S}(x) \cdot \mathbf{S}(y) \rightarrow[\mathbf{S}(x) \cdot \mathbf{S}(y)]_{h},
$$

with

$$
\begin{aligned}
{[\mathbf{S}(x) \cdot \mathbf{S}(y)]_{h}=} & \mathrm{e}^{\left(y_{0}-x_{0}\right) h} S_{-}(x) S_{+}(y)+\mathrm{e}^{-\left(y_{0}-x_{0}\right) h} S_{+}(x) S_{-}(y)+\sum_{a=3}^{n} S_{a}(x) S_{a}(y) \\
= & \mathbf{S}(x) \cdot \mathbf{S}(y)+i \sinh \left(\left(y_{0}-x_{0}\right) h\right)\left[S_{1}(x) S_{2}(y)-S_{2}(x) S_{1}(y)\right] \\
& +\left\{\cosh \left(\left(y_{0}-x_{0}\right) h\right)-1\right\}\left[S_{1}(x) S_{1}(y)+S_{2}(x) S_{2}(y)\right]
\end{aligned}
$$

where $S_{ \pm}=\frac{1}{\sqrt{2}}\left(S_{1} \pm i S_{2}\right)$. This gives an additional $h$-dependent part $\mathcal{A}_{h}$ to the total action of the form

$$
\mathcal{A}_{h}=Z_{4} A_{2 h}+\sum_{i=1}^{5} \frac{g_{4}^{(i)}}{4} A_{4 h}^{(i)} \text {. }
$$

Further writing

$$
\begin{aligned}
& A_{2 h}=i h B_{2}+h^{2} C_{2}+\ldots, \\
& A_{4 h}^{(i)}=i h B_{4}^{(i)}+h^{2} C_{4}^{(i)}+\ldots,
\end{aligned}
$$

we have

$$
\begin{aligned}
& B_{2}=-\frac{1}{g_{0}^{2}} \sum_{x}\left[S_{1}(x) S_{2}(x+\hat{0})-S_{2}(x) S_{1}(x+\hat{0})\right], \\
& C_{2}=-\frac{1}{2 g_{0}^{2}} \sum_{x}\left[S_{1}(x) S_{1}(x+\hat{0})+S_{2}(x) S_{2}(x+\hat{0})\right],
\end{aligned}
$$

and the terms $B_{4}^{(i)}, C_{4}^{(i)}$ are given in appendix A.

\footnotetext{
${ }^{2}$ For clarity we re-introduced here the lattice spacing.
} 
The $h$-dependent part of the free energy $f_{h}$ defined by:

$$
\mathrm{e}^{-V f_{h}}=\left\langle\mathrm{e}^{-\mathcal{A}_{h}}\right\rangle_{\mathcal{A}}=1-\left\langle\mathcal{A}_{h}\right\rangle_{\mathcal{A}}+\frac{1}{2}\left\langle\mathcal{A}_{h}^{2}\right\rangle_{\mathcal{A}}+\ldots
$$

giving up to the order $h^{2}$ :

$$
V f_{h}=\left\langle\mathcal{A}_{h}\right\rangle_{\mathcal{A}}-\frac{1}{2}\left\langle\mathcal{A}_{h}^{2}\right\rangle_{\mathcal{A}}+\frac{1}{2}\left\langle\mathcal{A}_{h}\right\rangle_{\mathcal{A}}^{2}+\ldots
$$

For finite volumes the $\lim _{h \rightarrow 0}\left(f_{h} / h^{2}\right)$ exists; we define the corresponding susceptibility $\chi$ by

$$
\chi \equiv-2 \lim _{h \rightarrow 0}\left(f_{h} / h^{2}\right) .
$$

Now

$$
\left\langle B_{2}\right\rangle_{\mathcal{A}}=0=\left\langle B_{4}^{(i)}\right\rangle_{\mathcal{A}} \quad \forall i
$$

so we have

$$
\chi=-2 \sum_{s=1}^{5} F_{s},
$$

with

$$
\begin{aligned}
& F_{1}=Z_{4} \frac{1}{V}\left\langle C_{2}\right\rangle_{\mathcal{A}}, \\
& F_{2}=\frac{1}{2} Z_{4}^{2} \frac{1}{V}\left\langle B_{2}^{2}\right\rangle_{\mathcal{A}}, \\
& F_{3}=\sum_{i=1}^{5} \frac{g_{4}^{(i)}}{4} \frac{1}{V}\left\langle C_{4}^{(i)}\right\rangle_{\mathcal{A}}, \\
& F_{4}=Z_{4} \sum_{i=1}^{5} \frac{g_{4}^{(i)}}{4} \frac{1}{V}\left\langle B_{2} B_{4}^{(i)}\right\rangle_{\mathcal{A}}, \\
& F_{5}=\frac{1}{2} \sum_{i j} \frac{g_{4}^{(i)}}{4} \frac{g_{4}^{(j)}}{4} \frac{1}{V}\left\langle B_{4}^{(i)} B_{4}^{(j)}\right\rangle_{\mathcal{A}} .
\end{aligned}
$$

In the following subsections where we compute the contributions $F_{s}$ we will use the fact that the total action $\mathcal{A}$ is invariant under global $\mathrm{O}(n)$ transformations of the spins, so that the expectation value of any observable is equal to the expectation value of its average over $\mathrm{O}(n)$ rotations $\Omega:^{3}$

$$
\langle\mathcal{O}[S]\rangle_{\mathcal{A}}=\left\langle[\mathcal{O}[S]]_{\Omega}\right\rangle_{\mathcal{A}}
$$

For arbitrary spins $\mathbf{a}, \mathbf{b}, \mathbf{c}, \mathbf{d}$ we will use the following averages

$$
\left[a_{1} b_{1}+a_{2} b_{2}\right]_{\Omega}=\frac{2}{n}(\mathbf{a} \cdot \mathbf{b}),
$$

and

$$
\left[\left(a_{1} b_{2}-a_{2} b_{1}\right)\left(c_{1} d_{2}-c_{2} d_{1}\right)\right]_{\Omega}=\frac{2}{n(n-1)}[(\mathbf{a} \cdot \mathbf{c})(\mathbf{b} \cdot \mathbf{d})-(\mathbf{a} \cdot \mathbf{d})(\mathbf{b} \cdot \mathbf{c})] .
$$

\footnotetext{
${ }^{3}$ Recall that we consider finite volume where no spontaneous symmetry breaking occurs.
} 


\subsubsection{Computation of $F_{1}$ up to $\mathrm{O}\left(g_{0}^{2}\right)$}

Averaging over the rotations (using (2.62)) we have

$$
\frac{1}{V}\left[C_{2}\right]_{\Omega}=-\frac{1}{n g_{0}^{2}}+\frac{1}{2 n g_{0}^{2}} U,
$$

where

$$
U=\frac{1}{V} \sum_{x} \partial_{0} \mathbf{S}(x) \cdot \partial_{0} \mathbf{S}(x) .
$$

This has the perturbative expansion

$$
U=g_{0}^{2} U_{1}+g_{0}^{4} U_{2}+\ldots
$$

with

$$
\begin{aligned}
U_{1} & =\frac{1}{V} \sum_{x} \partial_{0} \vec{\pi}(x) \cdot \partial_{0} \vec{\pi}(x), \\
U_{2} & =\frac{1}{4 V} \sum_{x}\left[\partial_{0} \vec{\pi}(x)^{2}\right]^{2} .
\end{aligned}
$$

Expanding (2.56) in a perturbative series

$$
F_{1}=-\frac{1}{n g_{0}^{2}}+\sum_{r=0}^{\infty} F_{1, r} g_{0}^{2 r}
$$

we have at leading orders

$$
F_{1,0}=\frac{1}{2 n}\left\langle U_{1}\right\rangle_{0}
$$

and

$$
F_{1,1}=\frac{1}{2 n}\left[-2 Z_{4,2}+\left\langle U_{2}\right\rangle_{0}-\left\langle U_{1} A_{2,1}\right\rangle_{0}^{c}-\sum_{i=1}^{5} \frac{g_{4,0}^{(i)}}{4}\left\langle U_{1} A_{4,1}^{(i)}\right\rangle_{0}^{c}\right],
$$

where the superscript ${ }^{c}$ in $\langle\ldots\rangle_{0}^{c}$ means the connected part. The correlation functions appearing in (2.69), (2.70) are given in appendix C.1 yielding

$$
F_{1,0}=\frac{n_{1}}{2 n} I_{11},
$$

and

$$
\begin{aligned}
F_{1,1}= & \frac{n_{1}}{2 n}\left[-\frac{2}{n_{1}} Z_{4,2}+I_{11}\left\{I_{10}-\frac{1}{4} I_{11}\right\}-\mathcal{F}_{1}+\left(1-\frac{n_{1}}{V}\right) I_{21}\right. \\
& \left.-\frac{g_{4,0}^{(1)}}{2} I_{01}-\frac{g_{4,0}^{(5)}}{2}\left\{J_{21}-\frac{1}{d+2}\left(2 I_{01}+\mathcal{F}_{4}\right)\right\}\right] .
\end{aligned}
$$

Here $I_{n m}, J_{n m}, \mathcal{F}_{1}, \mathcal{F}_{4}$ are momentum sums defined in equations (B.1), (B.2), (B.4), (B.6), and (B.9) respectively.

\subsubsection{Computation of $F_{2}$ up to $\mathrm{O}\left(g_{0}^{2}\right)$}

Averaging over the rotations one has, using (2.63),

$$
\frac{1}{V}\left[B_{2}^{2}\right]_{\Omega}=\frac{4}{n n_{1} g_{0}^{4}} W
$$


where $W$ is given by

$$
W=\frac{1}{V} \sum_{x y} \nabla_{0} \mathbf{S}(x) \cdot \nabla_{0} \mathbf{S}(y)[\mathbf{S}(x) \cdot \mathbf{S}(y)-1],
$$

where $\nabla_{0}=\frac{1}{2}\left(\partial_{0}+\partial_{0}^{*}\right)$ is the symmetric derivative. $W$ has a perturbative expansion

$$
W=g_{0}^{4} W_{2}+g_{0}^{6} W_{3}+\ldots
$$

with (to the order we need)

$$
\begin{aligned}
& W_{2}=\frac{1}{V} \sum_{x y}\left[\nabla_{0} \vec{\pi}(x) \cdot \nabla_{0} \vec{\pi}(y)\right] \vec{\pi}(x) \cdot \vec{\pi}(y), \\
& W_{3}=\frac{1}{2 V} \sum_{x y}\left[\nabla_{0} \vec{\pi}(x) \cdot \nabla_{0} \vec{\pi}(y)\right] \vec{\pi}(x)^{2} \vec{\pi}(y)^{2} .
\end{aligned}
$$

Expanding (2.57) in a perturbative series

$$
F_{2}=\sum_{r=0}^{\infty} F_{2, r} g_{0}^{2 r}
$$

we have at leading order

$$
F_{2,0}=\frac{2}{n n_{1}}\left\langle W_{2}\right\rangle_{0}
$$

and at next order

$$
F_{2,1}=\frac{2}{n n_{1}}\left[\left\langle W_{3}\right\rangle_{0}-\left\langle W_{2} A_{2,1}\right\rangle_{0}^{c}-\sum_{i=1}^{5} \frac{g_{4,0}^{(i)}}{4}\left\langle W_{2} A_{4,1}^{(i)}\right\rangle_{0}^{c}\right] .
$$

The correlation functions appearing in (2.79) and (2.80) are computed in appendix C.2 yielding

$$
F_{2,0}=\frac{2\left(n_{1}-1\right)}{n}\left[I_{21}-\frac{1}{4} I_{22}\right]
$$

and

$$
\begin{aligned}
F_{2,1}= & \frac{1}{n} W_{3 c}+\frac{2\left(n_{1}-1\right)}{n}\left[W_{3 a}-2 \mathcal{F}_{2}+\mathcal{F}_{3}+2\left(1-\frac{n_{1}}{V}\right)\left\{I_{31}-\frac{1}{4} I_{32}\right\}\right. \\
& \left.-g_{4,0}^{(1)}\left(I_{11}-\frac{1}{4} I_{12}\right)-g_{4,0}^{(5)}\left(J_{31}-\frac{1}{4} J_{32}-\frac{1}{d+2}\left\{2 I_{11}-\frac{1}{2} I_{12}+\mathcal{F}_{5}\right\}\right)\right],
\end{aligned}
$$

where $\mathcal{F}_{2}, \mathcal{F}_{3} \mathcal{F}_{5}$ are defined in (B.7), (B.8), (B.10), and $W_{3 a}, W_{3 c}$ are defined through

$$
\begin{aligned}
& W_{3 a}=-\sum_{x} G(x)^{2} \nabla_{0}^{2} G(x), \\
& W_{3 c}=\sum_{x} \nabla_{0} G(x)\left[\left(\partial_{0} G(x)\right)^{2}-\left(\partial_{0}^{*} G(x)\right)^{2}\right]=-\frac{1}{6} \sum_{x}\left[\square_{0} G(x)\right]^{3} .
\end{aligned}
$$




\subsubsection{Summary}

The computation of the leading contributions to $F_{3}, F_{4}, F_{5}$ follows similar steps as in the subsections above and details are presented in appendices C.3-C.5. Summarizing our results so far, the susceptibility with standard lattice regularization is given by

$$
\chi=\frac{2}{n g_{0}^{2}}\left(1+\bar{R}_{1} g_{0}^{2}+\bar{R}_{2} g_{0}^{4}+\ldots\right),
$$

with

$$
\bar{R}_{1}=-\frac{n_{1}}{2} I_{11}-2\left(n_{1}-1\right)\left(I_{21}-\frac{1}{4} I_{22}\right),
$$

and

$$
\begin{aligned}
\bar{R}_{2}= & \bar{R}_{2}^{(a)}+\bar{R}_{2}^{(b)} \\
\bar{R}_{2}^{(a)}= & -\frac{1}{2} n_{1}\left[I_{11}\left\{I_{10}-\frac{1}{4} I_{11}\right\}-\mathcal{F}_{1}+\left(1-\frac{n_{1}}{V}\right) I_{21}\right] \\
& -W_{3 c}-2\left(n_{1}-1\right)\left[W_{3 a}-2 \mathcal{F}_{2}+\mathcal{F}_{3}+2\left(1-\frac{n_{1}}{V}\right)\left\{I_{31}-\frac{1}{4} I_{32}\right\}\right],
\end{aligned}
$$

and

$$
\bar{R}_{2}^{(b)}=\sum_{i=1}^{5} g_{4,0}^{(i)} G^{(i)},
$$

with

$$
\begin{aligned}
G^{(1)}= & -\frac{1}{2} \bar{c}^{(1)}+2 I_{11}-\frac{1}{2} I_{12}+n_{1}\left[I_{00}-\frac{1}{4} I_{01}\right] \\
G^{(2)}= & -\frac{1}{2} \bar{c}^{(2)}+2 I_{11}+n_{1} I_{00} \\
G^{(3)}= & -\frac{1}{2} \bar{c}^{(3)}+I_{00}+\left(n_{1}+1\right) I_{11} \\
G^{(4)}= & -\frac{1}{2} \bar{c}^{(4)}-\frac{\left(n_{1}+2\right)}{(d+2)}\left[I_{00}-d I_{11}\right] \\
G^{(5)}= & -\frac{1}{2} \bar{c}^{(5)}-\frac{2}{(d+2)}\left\{2 I_{11}-\frac{1}{2} I_{12}+n_{1}\left[I_{00}-\frac{1}{4} I_{01}\right]\right\} \\
& -\frac{n_{1}}{(d+2)}\left[-(d+1)\left\{3 I_{11}-I_{12}\right\}+\mathcal{F}_{6}-\frac{1}{4}(d+2) J_{21}+\frac{1}{4} \mathcal{F}_{4}\right] \\
& +\left(n_{1}-1\right)\left\{2 J_{31}-\frac{1}{2} J_{32}-\frac{1}{(d+2)}\left[2 \mathcal{F}_{5}+(d+1)\left(4 I_{22}-I_{23}\right)-2 \mathcal{F}_{7}\right]\right\} .
\end{aligned}
$$

A check of (2.88) for the special case of $n=2$ is given in appendix D.

\subsection{Renormalization on the lattice}

The renormalization procedure depends on the dimension; in the following we will consider the cases $d=2,3,4$. 


\subsubsection{Case $d=2$}

For $d=2$ the theory is renormalizable so we can set the 4-derivative couplings $g_{4}^{(i)}$ to zero. As is well known the theory is asymptotically free.

A renormalized "minimal" lattice coupling $g_{\text {latt }}(\mu)$ is defined through

$$
\frac{1}{g_{0}^{2}}=\frac{1}{g_{\text {latt }}^{2}(\mu)}-b_{0} \ln (a \mu)-b_{1} \ln (a \mu) g_{\text {latt }}^{2}(\mu)+\ldots
$$

where $b_{0}, b_{1}$ are the universal 1-, and 2-loop coefficients of the $\beta$-function [14, 15]:

$$
b_{0}=\frac{n-2}{2 \pi}, \quad b_{1}=\frac{n-2}{4 \pi^{2}} .
$$

In the continuum limit

$$
\begin{aligned}
& I_{11}=\frac{1}{2}+\mathrm{O}\left(a^{2}\right), \\
& I_{21}=\frac{1}{4 \pi} \ln \left(L_{s} / a\right)+I_{21 ; 0}(\ell)+\mathrm{O}\left(a^{2}\right), \\
& I_{22}=\frac{1}{2}-\frac{1}{2 \pi}+\mathrm{O}\left(a^{2}\right) .
\end{aligned}
$$

The coefficients $I_{n m ; r}$ appearing in the large $L_{s} / a$ expansion of $I_{n m}$ are considered in [9].

So

$$
\bar{R}_{1}=-b_{0} \ln \left(L_{s} / a\right)+\bar{r}_{1}+\mathrm{O}\left(a^{2}\right)
$$

with

$$
\bar{r}_{1}=-\frac{1}{4}-2(n-2)\left[I_{21 ; 0}(\ell)+\frac{1}{8 \pi}\right] .
$$

Next

$$
\begin{aligned}
I_{10}= & \frac{1}{2 \pi} \ln \left(L_{s} / a\right)+I_{10 ; 0}(\ell)+\mathrm{O}\left(a^{2}\right) \\
I_{32}= & \frac{3}{16 \pi} \ln \left(L_{s} / a\right)+I_{32 ; 0}(\ell)+\mathrm{O}\left(a^{2}\right) \\
\frac{1}{V} I_{31}= & I_{31 ;-2}(\ell)+\mathrm{O}\left(a^{2}\right) \\
\mathcal{F}_{1}= & \frac{1}{2 \pi} \ln \left(L_{s} / a\right)+I_{21 ; 0}(\ell)+\frac{1}{2} I_{10 ; 0}(\ell)-\frac{1}{8}+\mathrm{O}\left(a^{2}\right), \\
\mathcal{F}_{2}-I_{31}= & \frac{1}{8 \pi^{2}} \ln ^{2}\left(L_{s} / a\right)+\frac{1}{2 \pi}\left\{I_{21 ; 0}(\ell)+\frac{1}{2} I_{10 ; 0}(\ell)+\frac{1}{8 \pi}-\frac{11}{32}\right\} \ln \left(L_{s} / a\right) \\
& +\mathcal{F}_{2 ; 0}+\mathrm{O}\left(a^{2}\right), \\
\mathcal{F}_{2 ; 0}= & {\left[I_{10 ; 0}(\ell)-\frac{1}{4}\right]\left\{I_{21 ; 0}(\ell)-\frac{1}{8}+\frac{1}{8 \pi}\right\}-I_{31 ;-2}(\ell)-\frac{1}{4} I_{32 ; 0}(\ell), } \\
W_{3 a}= & \frac{1}{8 \pi^{2}} \ln ^{2}\left(L_{s} / a\right)+W_{3 a ; 0 x}(\ell) \ln \left(L_{s} / a\right)+W_{3 a ; 0}(\ell)+\mathrm{O}\left(a^{2}\right), \\
W_{3 c}= & \frac{1}{48}+\mathrm{O}\left(a^{2}\right) .
\end{aligned}
$$

So

$$
\bar{R}_{2}^{(a)}=-b_{1} \ln \left(L_{s} / a\right)+\bar{r}_{2}+\mathrm{O}\left(a^{2}\right)
$$


with

$$
\begin{aligned}
\bar{r}_{2}= & -\frac{5}{96}+4(n-2)^{2} I_{31 ;-2}(\ell) \\
& -2(n-2)\left[W_{3 a ; 0}(\ell)+\frac{1}{64}-2\left\{I_{10 ; 0}(\ell)-I_{21 ; 0}(\ell)-\frac{1}{8}-\frac{1}{8 \pi}\right\}\left\{I_{21 ; 0}(\ell)-\frac{1}{8}+\frac{1}{8 \pi}\right\}\right],
\end{aligned}
$$

where we have used the relation

$$
W_{3 a ; 0 x}(\ell)=\frac{1}{2 \pi}\left[I_{10 ; 0}(\ell)-\frac{1}{4}+\frac{1}{4 \pi}\right] .
$$

We thus obtain in the continuum limit:

$$
\begin{aligned}
\chi & =\frac{2}{n g_{\text {latt }}^{2}(\mu)}\left\{1+\left[-b_{0} \ln \left(\mu L_{s}\right)+\bar{r}_{1}\right] g_{\text {latt }}^{2}(\mu)+\left[-b_{1} \ln \left(\mu L_{s}\right)+\bar{r}_{2}\right] g_{\text {latt }}^{4}(\mu)+\ldots\right\} \\
& =\frac{2}{n g_{\text {latt }}^{2}\left(1 / L_{s}\right)}\left\{1+\bar{r}_{1} g_{\text {latt }}^{2}\left(1 / L_{s}\right)+\bar{r}_{2} g_{\text {latt }}^{4}\left(1 / L_{s}\right)+\ldots\right\}
\end{aligned}
$$

which is interpreted as an expansion in the running lattice coupling $g_{\text {latt }}\left(1 / L_{s}\right)$, the expansion being sensible only for physically small box size $L_{s}$.

\subsubsection{Case $d=3$}

For $d=3$ we set $g_{0}^{2}=1 /\left(\rho_{0} a\right)$, where $\rho_{0}$ is the bare spin stiffness, and define a renormalized coupling $\rho$ as in [7] through

$$
\frac{1}{\rho_{0}}=\frac{1}{\rho}\left(1+\frac{b_{1}}{\rho a}+\frac{b_{2}}{\rho^{2} a^{2}}+\ldots\right) .
$$

Then we have

$$
\chi=\frac{2 \rho}{n}\left(1+\frac{1}{\rho a} \hat{R}_{1}+\frac{1}{\rho^{2} a^{2}} \hat{R}_{2}+\ldots\right)
$$

with

$$
\begin{aligned}
& \hat{R}_{1}=\bar{R}_{1}-b_{1}, \\
& \hat{R}_{2}=\bar{R}_{2}-b_{2}+b_{1}^{2} .
\end{aligned}
$$

From [9] for $d=3 \bar{R}_{1}$ has a large $L_{s} / a$ expansion of the form

$$
\bar{R}_{1}=-\frac{1}{6}-(n-2) I_{10 ; 0}-2(n-2) I_{21 ; 1}(\ell) \frac{a}{L_{s}}+\ldots
$$

with $I_{10 ; 0}=0.252731009859$, where the large $L_{s} / a$ expansion of $X_{A}$ is given by $X_{A}=$ $\sum_{r=r_{0}} X_{A ; r}\left(a / L_{s}\right)^{r}$. So for renormalization at leading order we need

$$
b_{1}=-\frac{1}{6}-(n-2) I_{10 ; 0} .
$$

After choosing the $\bar{c}^{(i)}$ appropriately the terms in $\hat{R}_{2}$ coming from $\bar{R}_{2}^{(b)}$ are of order $a^{3} / L_{s}^{3},{ }^{4}$ so the continuum limit is determined only by $\bar{R}_{2}^{(a)}$.

\footnotetext{
${ }^{4}$ The couplings of the 4 -derivative interactions in $d=3$ have dimension in the continuum formulation.
} 
Further $\bar{R}_{2}^{(a)}$ has a large $L_{s} / a$ expansion for $d=3$ of the form

$$
\bar{R}_{2}^{(a)}=\bar{R}_{2 ; 0}^{(a)}+\bar{R}_{2 ; 1}^{(a)} \frac{a}{L_{s}}+\bar{R}_{2 ; 2}^{(a)} \frac{a^{2}}{L_{s}^{2}}+\ldots
$$

so renormalization requires

$$
b_{2}-b_{1}^{2}=\bar{R}_{2 ; 0}
$$

which gives

$$
b_{2}=b_{20}+b_{21} n_{1}+b_{22} n_{1}^{2},
$$

with coefficients independent of $\ell^{5}$

$$
\begin{aligned}
& b_{20}=2 W_{3 a ; 0}-W_{3 c ; 0}=0.0102138509611 \\
& b_{21}=\frac{1}{72}-2 W_{3 a ; 0}-I_{10 ; 0}^{2}=-0.0659002864141, \\
& b_{22}=I_{10 ; 0}^{2}=0.0638729633447 .
\end{aligned}
$$

Further we need

$$
0=\bar{R}_{2 ; 1}^{(a)}=2(n-2)\left[-W_{3 a ; 1}+\left\{I_{10 ; 0}-\frac{1}{6}\right\} I_{10 ; 1}\right]
$$

which we have verified numerically to high precision for $\ell=1$ and $\ell=2$ [9].

So finally we have for $d=3$ in the continuum limit:

$$
\chi=\frac{2 \rho}{n}\left(1-\frac{1}{\rho L_{s}} 2(n-2) I_{21 ; 1}(\ell)+\frac{1}{\rho^{2} L_{s}^{2}} \bar{R}_{2 ; 2}^{(a)}(\ell)+\ldots\right)
$$

with

$$
\bar{R}_{2 ; 2}^{(a)}=2(n-2)\left\{-W_{3 a ; 2}+2 I_{21 ; 1}\left(I_{10 ; 1}-I_{21 ; 1}\right)+\frac{2}{\ell}(n-2) I_{31 ;-1}\right\} .
$$

This result for the susceptibility agrees with $(2.29)$ of $[6]{ }^{6}$ e.g.:

$$
\bar{R}_{2 ; 2}^{(a)}(\ell)=\left\{\begin{array}{cc}
-0.00920015939-0.007071685928(n-2), & \text { for } \ell=1, \\
0.01560323409-0.01338624986(n-2), & \text { for } \ell=2 .
\end{array}\right.
$$

\subsubsection{Case $d=4$}

For $d=4$ we set $g_{0}^{2}=1 /\left(F_{0}^{2} a^{2}\right)$ and define a renormalized coupling $F$ (the pion decay constant in chiral PT in the chiral limit) through

$$
\frac{1}{F_{0}^{2}}=\frac{1}{F^{2}}\left(1+\frac{b_{1}}{F^{2} a^{2}}+\frac{b_{2}}{F^{4} a^{4}}+\mathrm{O}\left(1 /(F a)^{6}\right)\right) .
$$

\footnotetext{
${ }^{5}$ Note $4 I_{21 ; 0}-I_{22 ; 0}=2 I_{10 ; 0}-1 / d$.

${ }^{6}$ With the identification of the notation used there (on the lhs): $\beta_{1}=-I_{10 ; 1}, \beta_{2}=I_{20 ;-1}, \tilde{\beta}_{1}=$ $-6 I_{21 ; 1}, \quad \tilde{\beta}_{2}=(12 / \ell) I_{31 ;-1}, \quad \psi=W_{3 a ; 2}-2 I_{10 ; 1} I_{21 ; 1}$.
} 
After renormalization we have

$$
\chi=\frac{2 F^{2}}{n}\left(1+\frac{1}{F^{2} a^{2}} \hat{R}_{1}+\frac{1}{F^{4} a^{4}} \hat{R}_{2}+\ldots\right),
$$

with

$$
\begin{aligned}
& \hat{R}_{1}=\bar{R}_{1}-b_{1}, \\
& \hat{R}_{2}=\bar{R}_{2}-b_{2}+b_{1}^{2} .
\end{aligned}
$$

To cancel the $1 / a^{2}$ terms in $\hat{R}_{1} / a^{2}$ one should require

$$
\begin{aligned}
b_{1} & =-\frac{1}{8}-(n-2) I_{10 ; 0} \\
& =0.029933390231060214084-0.15493339023106021408 n_{1} .
\end{aligned}
$$

This agrees with the result in [7].

Next since $I_{11 ; 2}=0=I_{22 ; 2}$ after renormalization we obtain

$$
\lim _{a \rightarrow 0}\left[a^{-2} \hat{R}_{1}\right]=-\frac{2(n-2)}{L_{s}^{2}} I_{21 ; 2} .
$$

$\hat{R}_{2} / a^{4}$ has divergent terms proportional to $1 / a^{4}, 1 / a^{2}$ and $\log (a)$. First we recall that the subtraction coefficients $c^{(i)}$ are used to cancel the leading, $1 / a^{4}$ contributions of the corresponding operators. In leading order $\bar{c}^{(i)}$ (defined in (2.30)) is fixed by requiring

$$
\lim _{a / L_{s} \rightarrow 0} G^{(i)}=0
$$

where $G^{(i)}$ are the coefficients in (2.89), which leads to

$$
\begin{aligned}
& \bar{c}^{(1)}=n-I_{12 ; 0}=n-0.7066242375215119838793013966, \\
& \bar{c}^{(2)}=2 n-1, \\
& \bar{c}^{(3)}=\frac{1}{2} n+2, \\
& \bar{c}^{(4)}=0, \\
& \bar{c}^{(5)}=-0.030936190551839592713 n-0.032327591899970596813 .
\end{aligned}
$$

In [7] the overall sign of $\bar{c}^{(i)}$ was wrong and we also disagree here with sign of the constant term in $\bar{c}^{(5)}$.

Demanding the absence of the $1 / a^{4}$ singularity in $\hat{R}_{2} / a^{4}$ determines the second order coefficient

$$
\begin{aligned}
b_{2} & =I_{10 ; 0}^{2}(n-2)^{2}+\left(I_{10 ; 0}^{2}+\frac{1}{128}-2 W_{3 a ; 0}\right)(n-2)+\frac{1}{128}-W_{3 c ; 0} \\
& =0.024004355408(n-2)^{2}+0.028115270716(n-2)+0.005536500909 .
\end{aligned}
$$

This agrees with [7]. 
With the values for $\bar{c}^{(i)}$ above $G^{(i)}=\mathrm{O}\left(a^{4} / L_{s}^{4}\right) \forall i$. It follows that the $1 / a^{2}$ contribution to $\hat{R}_{2} / a^{4}$ has no more free parameters and should vanish identically. This requires the relation

$$
W_{3 a ; 2}=\left(I_{10 ; 0}-\frac{1}{8}\right) I_{10 ; 2}
$$

which indeed holds numerically (see [9]).

As for the renormalization of the 4-derivative couplings one has

$$
g_{4,0}^{(i)}=k^{(i)} \log \left(a M_{i}\right), \quad i=1,2,3,
$$

while for $i=4,5$ they are not renormalized to this order.

Moreover it is easy to check that after choosing the $\bar{c}^{(i)}$ as above ${ }^{7}$

$$
G^{(1)}=G^{(2)}=\frac{a^{4}}{L_{s}^{4}}\left[2 I_{11 ; 2}-\frac{n_{1}}{\ell}\right]+\mathrm{O}\left(a^{6} / L_{s}^{6}\right)
$$

so that the part of $\hat{R}_{2}$ contributing in the continuum limit depends on $g_{4,0}^{(1)}, g_{4,0}^{(2)}$ only through their sum $g_{4,0}^{(1)}+g_{4,0}^{(2)}$, consistent with our general argument on the redundancy of the 4derivative operators in subsection 2.1. In the following we shall use this redundancy to set $g_{4}^{(1)}=0$.

The cancellation of the $\ln \left(L_{s} / a\right)$ terms requires a relation for the coefficient of the $N^{-4} \ln N$ term in $W_{3 a}$,

$$
W_{3 a ; 4 x}=\frac{1}{48 \pi^{2}}\left(10 I_{11 ; 4}+\frac{1}{\ell}\right)
$$

which is satisfied numerically to high precision. Then the coefficients of the logarithmic terms of $g_{4,0}^{(2)}$ and $g_{4,0}^{(3)}$ are fixed as:

$$
\begin{aligned}
& -4 \pi^{2} k^{(2)}=w_{1}=\frac{n}{2}-\frac{5}{3}, \\
& -4 \pi^{2} k^{(3)}=w_{2}=\frac{2}{3},
\end{aligned}
$$

agreeing with refs. [7] and [2].

Noting the relation

$$
W_{3 c ; 4}=-\frac{1}{8}\left(4 I_{10 ; 0}-1\right) I_{11 ; 4}
$$

which is satisfied by the numerical values in [9], and inserting eqs. (4.22) and (4.23) one obtains the continuum limit of $\hat{R}_{2} / a^{4}$ :

$$
L_{s}^{4} \lim _{a \rightarrow 0} \frac{\hat{R}_{2}}{a^{4}}=\widehat{H}_{0}-H_{2} \frac{w_{1}}{4 \pi^{2}} \ln \left(M_{2} L_{s}\right)-H_{3} \frac{w_{2}}{4 \pi^{2}} \ln \left(M_{3} L_{s}\right)+H_{4} g_{4,0}^{(4)}+H_{5} g_{4,0}^{(5)},
$$

\footnotetext{
${ }^{7}$ Note $I_{12 ; 4}=0$.
} 
where

$$
\begin{aligned}
& H_{2}=-\frac{n-1}{\ell}+2 I_{11 ; 4}, \\
& H_{3}=-\frac{1}{\ell}+n I_{11 ; 4}, \\
& H_{4}=\frac{1}{6}(n+1)\left(\frac{1}{\ell}+4 I_{11 ; 4}\right), \\
& H_{5}=\frac{n-1}{2 \ell}+(3 n-4) I_{11 ; 4}+2(n-2)\left(J_{31 ; 4}-2 I_{22 ; 4}\right),
\end{aligned}
$$

and

$$
\widehat{H}_{0}=-(n-2) \widehat{w}+\widehat{w}^{\prime} I_{11 ; 4}+\widehat{w}^{\prime \prime} \frac{1}{\ell},
$$

with

$$
\begin{aligned}
\widehat{w} & =-2\left(I_{10 ; 0}-\frac{1}{8}\right) I_{10 ; 4}+2 W_{3 a ; 4}-4\left(I_{10 ; 2}-I_{21 ; 2}\right) I_{21 ; 2} \\
\widehat{w}^{\prime} & =\frac{2}{3}(n-2)\left(I_{33 ; 0}-4 I_{32 ; 0}\right)+\frac{2}{3}\left(n-\frac{3}{4}\right) I_{10 ; 0}-\frac{1}{48}(5 n-1) \\
\widehat{w}^{\prime \prime} & =\frac{1}{24}\left(3 n^{2}+n-12\right) I_{10 ; 0}-(n-2)\left\{\left(n-\frac{4}{3}\right) I_{32 ; 0}-\frac{1}{6} I_{33 ; 0}+\frac{1}{24}\right\}+4(n-2)^{2} I_{31 ; 0} .
\end{aligned}
$$

Note that the coefficient of $g_{4,0}^{(4)}$ in eq. (2.144) vanishes for the hyper-cubic case, $\ell=1$.

\section{The free energy with dimensional regularization}

In this section we work in a continuum volume $V=L_{t} \times L_{s}^{d_{s}}, d_{s}=d-1$. Again the dynamical variables are spins $S_{a}(x), a=1, \ldots, n$ of unit length $\mathbf{S}(x)^{2}=1$ with periodic boundary conditions in all directions. We will dimensionally regularize by adding $q$ extra compact dimensions of size $\widehat{L}$ (also with pbc) and analytically continue the resulting loop formulae to $q=-2 \epsilon$. We define $D=d+q, V_{D}=V \widehat{L}^{q}$. We denote aspect ratio of the extra dimensions by $\hat{\ell} \equiv \widehat{L} / L_{s}$. It is advantageous to treat these extra dimensions with a different size, since an extra check of the calculation is provided by the requirement that physical quantities are independent of this choice.

Many of the formulae are similar to those with lattice regularization and we will duplicate many of the notations hoping that this will not lead to confusion.

\subsection{The effective action}

The effective action $\mathcal{A}$ is a sum over terms

$$
\mathcal{A}=A_{2}+A_{4}+\ldots
$$

where $A_{2 r}$ has $2 r$ derivatives. $A_{2}$ is simply given by

$$
A_{2}=\frac{1}{2 g_{0}^{2}} \int_{x} \sum_{\mu} \partial_{\mu} \mathbf{S}(x) \cdot \partial_{\mu} \mathbf{S}(x) .
$$


The four derivative terms are

$$
A_{4}=\sum_{i=2,3} \frac{g_{4}^{(i)}}{4} A_{4}^{(i)},
$$

where (we use redundancy immediately here to set $g_{4}^{(1)}=0$ ),

$$
\begin{aligned}
& A_{4}^{(2)}=\int_{x} \sum_{\mu \nu}\left[\partial_{\mu} \mathbf{S}(x) \cdot \partial_{\mu} \mathbf{S}(x)\right]\left[\partial_{\nu} \mathbf{S}(x) \cdot \partial_{\nu} \mathbf{S}(x)\right], \\
& A_{4}^{(3)}=\int_{x} \sum_{\mu \nu}\left[\partial_{\mu} \mathbf{S}(x) \cdot \partial_{\nu} \mathbf{S}(x)\right]\left[\partial_{\mu} \mathbf{S}(x) \cdot \partial_{\nu} \mathbf{S}(x)\right] .
\end{aligned}
$$

\subsection{Perturbative expansion}

After separating the zero mode and changing to $\vec{\pi}$ variables $\left(\mathbf{S}=\left(g_{0} \vec{\pi}, \sqrt{1-g_{0}^{2} \vec{\pi}^{2}}\right)\right)$

$$
A_{2, \mathrm{eff}}[\vec{\pi}]=A_{2}[\vec{\pi}]+A_{2, \mathrm{zero}}[\vec{\pi}]
$$

Note that the measure term is not present with dimensional regularization.

$$
A_{2, \text { zero }}[\vec{\pi}]=-n_{1} \ln \left(\frac{1}{V_{D}} \int_{x}\left(1-g_{0}^{2} \vec{\pi}(x)^{2}\right)^{\frac{1}{2}}\right) .
$$

$A_{2, \text { eff }}$ has a perturbative expansion

$$
A_{2, \mathrm{eff}}=A_{2,0}+g_{0}^{2} A_{2,1}+\mathrm{O}\left(g_{0}^{4}\right)
$$

where

$$
\begin{aligned}
A_{2,0} & =\frac{1}{2} \int_{x} \partial_{\mu} \vec{\pi}(x) \cdot \partial_{\mu} \vec{\pi}(x), \\
A_{2,1} & =A_{2,1}^{(a)}+A_{2,1}^{(b)}, \\
A_{2,1}^{(a)} & =\frac{n_{1}}{2 V_{D}} \int_{x} \vec{\pi}(x)^{2}, \\
A_{2,1}^{(b)} & =\frac{1}{8} \int_{x} \partial_{\mu}\left[\vec{\pi}(x)^{2}\right] \partial_{\mu}\left[\vec{\pi}(x)^{2}\right] .
\end{aligned}
$$

The total effective action has a perturbative expansion of the form

$$
\mathcal{A}=\sum_{r=0} \mathcal{A}_{r} g_{0}^{2 r}
$$

with

$$
\mathcal{A}_{r}=A_{2, r}, \quad r=0,1,
$$

since

$$
A_{4}^{(i)}=\mathrm{O}\left(g_{0}^{4}\right), \quad i=2,3 .
$$

The free 2-point function is given by

$$
\left\langle\pi_{a}(x) \pi_{b}(y)\right\rangle_{0}=\delta_{a b} G(x-y),
$$


with propagator

$$
G(x)=\frac{1}{V_{D}} \sum_{p}^{\prime} \frac{\mathrm{e}^{i p x}}{p^{2}},
$$

where the sum is over momenta $p_{\mu}=2 \pi n_{\mu} / L_{\mu}, n_{\mu} \in \mathbb{Z}$ and the prime on the sum means that $p=0$ is omitted.

\subsection{The chemical potential}

The chemical potential $h$ is introduced by the substitution:

$$
\partial_{0} \rightarrow \partial_{0}-h Q
$$

where $(Q S)_{1}=i S_{2},(Q S)_{2}=-i S_{1}$, and $(Q S)_{a}=0, a=3, \ldots, n$.

This gives an additional $h$-dependent part $\mathcal{A}_{h}$ to the total action of the form

$$
\mathcal{A}_{h}=A_{2 h}+\sum_{i=2,3} \frac{g_{4}^{(i)}}{4} A_{4 h}^{(i)} .
$$

Further writing

$$
\begin{aligned}
& A_{2 h}=i h B_{2}+h^{2} C_{2}+\ldots, \\
& A_{4 h}^{(i)}=i h B_{4}^{(i)}+h^{2} C_{4}^{(i)}+\ldots,
\end{aligned}
$$

we have

$$
\begin{aligned}
B_{2} & =-\frac{1}{g_{0}^{2}} \int_{x} j_{0}(x), \quad j_{\mu}(x)=S_{2}(x) \partial_{\mu} S_{1}(x)-S_{1}(x) \partial_{\mu} S_{2}(x), \\
C_{2} & =\frac{1}{2 g_{0}^{2}} \int_{x}[Q \mathbf{S}(x)]^{2} .
\end{aligned}
$$

For the operator 2:

$$
\begin{aligned}
& B_{4}^{(2)}=-4 \int_{x} \partial_{\mu} \mathbf{S}(x) \cdot \partial_{\mu} \mathbf{S}(x) j_{0}(x), \\
& C_{4}^{(2)}=-2 \int_{x}\left\{\partial_{\mu} \mathbf{S}(x) \cdot \partial_{\mu} \mathbf{S}(x)\left[S_{1}(x)^{2}+S_{2}(x)^{2}\right]+2\left[j_{0}(x)\right]^{2}\right\},
\end{aligned}
$$

and for the operator 3 :

$$
\begin{aligned}
& B_{4}^{(3)}=-4 \int_{x} \partial_{0} \mathbf{S}(x) \cdot \partial_{\mu} \mathbf{S}(x) j_{\mu}(x) \\
& C_{4}^{(3)}=-2 \int_{x}\left\{\partial_{0} \mathbf{S}(x) \cdot \partial_{0} \mathbf{S}(x)\left[S_{1}(x)^{2}+S_{2}(x)^{2}\right]+2\left[j_{0}(x)\right]^{2}+\left[j_{k}(x)\right]^{2}\right\} .
\end{aligned}
$$

The $h$-dependent part of the free energy $f_{h}$ is defined as in (2.51). Now

$$
\left\langle B_{2}\right\rangle_{\mathcal{A}}=0=\left\langle B_{4}^{(i)}\right\rangle_{\mathcal{A}} \quad \forall i,
$$


so we have

$$
\chi=-2 \sum_{s=1}^{5} F_{s},
$$

with

$$
\begin{aligned}
F_{1} & =\frac{1}{V_{D}}\left\langle C_{2}\right\rangle_{\mathcal{A}}, \\
F_{2} & =\frac{1}{2} \frac{1}{V_{D}}\left\langle B_{2}^{2}\right\rangle_{\mathcal{A}}, \\
F_{3} & =\sum_{i=2,3} \frac{g_{4}^{(i)}}{4} \frac{1}{V_{D}}\left\langle C_{4}^{(i)}\right\rangle_{\mathcal{A}}, \\
F_{4} & =\sum_{i=2,3} \frac{g_{4}^{(i)}}{4} \frac{1}{V_{D}}\left\langle B_{2} B_{4}^{(i)}\right\rangle_{\mathcal{A}}, \\
F_{5} & =\frac{1}{2} \sum_{i j} \frac{g_{4}^{(i)}}{4} \frac{g_{4}^{(j)}}{4} \frac{1}{V_{D}}\left\langle B_{4}^{(i)} B_{4}^{(j)}\right\rangle_{\mathcal{A}} .
\end{aligned}
$$

Averaging over the rotations we have simply

$$
\frac{1}{V_{D}}\left[C_{2}\right]_{\Omega}=-\frac{1}{n g_{0}^{2}},
$$

and

$$
F_{1}=-\frac{1}{n g_{0}^{2}}
$$

Next

$$
\frac{1}{V_{D}}\left[B_{2}^{2}\right]_{\Omega}=\frac{4}{n n_{1} g_{0}^{4}} W
$$

with $W$ given by

$$
W=\frac{1}{V_{D}} \int_{x y} \partial_{0} \mathbf{S}(x) \cdot \partial_{0} \mathbf{S}(y)[\mathbf{S}(x) \cdot \mathbf{S}(y)-1] .
$$

This has a perturbative expansion

$$
W=g_{0}^{4} W_{2}+g_{0}^{6} W_{3}+\ldots
$$

with

$$
\begin{aligned}
& W_{2}=\frac{1}{V_{D}} \int_{x y}\left[\partial_{0} \vec{\pi}(x) \cdot \partial_{0} \vec{\pi}(y)\right] \vec{\pi}(x) \cdot \vec{\pi}(y), \\
& W_{3}=\frac{1}{2 V_{D}} \int_{x y}\left[\partial_{0} \vec{\pi}(x) \cdot \partial_{0} \vec{\pi}(y)\right] \vec{\pi}(x)^{2} \vec{\pi}(y)^{2} .
\end{aligned}
$$

Expanding (3.31) in a perturbative series

$$
F_{2}=\sum_{r=0}^{\infty} F_{2, r} g_{0}^{2 r},
$$


we have at leading order

$$
\begin{aligned}
F_{2,0} & =\frac{2}{n n_{1}}\left\langle W_{2}\right\rangle_{0} \\
& =\frac{2(n-2)}{n} \int_{x}\left[\partial_{0} G(x)\right]^{2}=\frac{2(n-2)}{n} \bar{I}_{21},
\end{aligned}
$$

where dimensionally regularized sums $\bar{I}_{n m}$ are formally defined by

$$
\bar{I}_{n m}=\frac{1}{V} \sum_{p}^{\prime} \frac{\left(p_{0}^{2}\right)^{m}}{\left(p^{2}\right)^{n}} .
$$

Sums with $m=0$ were treated by Hasenfratz and Leutwyler [16]; we generalize their methods to sums with $m=1$ in [9].

At next order

$$
F_{2,1}=\frac{2}{n n_{1}}\left[\left\langle W_{3}\right\rangle_{0}-\left\langle W_{2} A_{2,1}\right\rangle_{0}^{c}\right]
$$

First

$$
\left\langle W_{3}\right\rangle_{0}=\frac{1}{2 V_{D}} \int_{x y}\left\langle\partial_{0} \vec{\pi}(x) \cdot \partial_{0} \vec{\pi}(y) \vec{\pi}(x)^{2} \vec{\pi}(y)^{2}\right\rangle_{0}=n_{1}(n-2) \bar{W}
$$

where

$$
\bar{W}=-\int_{x} G(x)^{2} \partial_{0}^{2} G(x) .
$$

This 2-loop function, the "massless sunset diagram", is calculated in detail in [9].

Next

$$
\begin{aligned}
\left\langle W_{2} A_{2,1}^{(a)}\right\rangle_{0}^{c} & =\frac{n_{1}}{2 V_{D}^{2}} \int_{x y u}\left\langle\partial_{0} \vec{\pi}(x) \cdot \partial_{0} \vec{\pi}(y)(\vec{\pi}(x) \cdot \vec{\pi}(y)) \vec{\pi}(u)^{2}\right\rangle_{0}^{c} \\
& =\frac{2 n_{1}^{2}(n-2)}{V_{D}^{2}} \int_{x y u} \partial_{0}^{x} \partial_{0}^{y} G(x-y) G(x-u) G(y-u) \\
& =\frac{2 n_{1}^{2}(n-2)}{V_{D}^{2}} \sum_{p}^{\prime} \frac{p_{0}^{2}}{\left(p^{2}\right)^{3}}=\frac{2 n_{1}^{2}(n-2)}{V_{D}} \bar{I}_{31}
\end{aligned}
$$

and

$$
\begin{aligned}
\left\langle W_{2} A_{2,1}^{(b)}\right\rangle_{0}^{c}= & \frac{1}{8 V_{D}} \int_{x y u}\left[\partial_{\mu}^{u} \partial_{\mu}^{v}\left\langle\partial_{0} \vec{\pi}(x) \cdot \partial_{0} \vec{\pi}(y)(\vec{\pi}(x) \cdot \vec{\pi}(y)) \vec{\pi}(u)^{2} \vec{\pi}(v)^{2}\right\rangle_{0}^{c}\right]_{v=u} \\
= & \frac{n_{1}(n-2)}{V_{D}} \int_{x y u} \partial_{\mu}^{u} \partial_{\mu}^{v}\left[2 \partial_{0}^{x} \partial_{0}^{y} G(x-y) G(x-u) G(y-v) G(u-v)\right. \\
& \left.-\partial_{0}^{x} G(x-u) \partial_{0}^{y} G(y-v) G(x-v) G(y-u)\right]\left.\right|_{v=u} \\
= & n_{1}(n-2) \frac{1}{V_{D}^{2}} \sum_{p q}^{\prime}\left[\frac{2 p_{0}^{2}(p-q)^{2}}{\left(p^{2}\right)^{3} q^{2}}+\frac{p_{0} q_{0}(p-q)^{2}}{\left(p^{2}\right)^{2}\left(q^{2}\right)^{2}}\right] \\
= & 2 n_{1}(n-2)\left[\bar{I}_{21} \bar{I}_{10}+\bar{I}_{31} \bar{I}_{00}-\bar{I}_{21}^{2}\right]
\end{aligned}
$$

Note that $\bar{I}_{00}=-\square G(0)=-1 / V_{D}$ since the dimensional regularization sets $\delta(0)=0$. 
For the contribution from the 4-derivative terms, averaging over rotations:

$$
\begin{aligned}
& {\left[C_{4}^{(2)}\right]_{\Omega}=-\frac{4}{n n_{1}} \int_{x}\left\{n_{1} \partial_{\mu} \mathbf{S}(x) \cdot \partial_{\mu} \mathbf{S}(x)+2 \partial_{0} \mathbf{S}(x) \cdot \partial_{0} \mathbf{S}(x)\right\}} \\
& {\left[C_{4}^{(3)}\right]_{\Omega}=-\frac{4}{n n_{1}} \int_{x}\left\{\partial_{\mu} \mathbf{S}(x) \cdot \partial_{\mu} \mathbf{S}(x)+n \partial_{0} \mathbf{S}(x) \cdot \partial_{0} \mathbf{S}(x)\right\}}
\end{aligned}
$$

So to first order perturbation theory

$$
F_{3,1}=\frac{4}{n}\left\{\frac{g_{4}^{(2)}}{4}\left[\frac{n_{1}}{V_{D}}-2 \bar{I}_{11}\right]+\frac{g_{4}^{(3)}}{4}\left[\frac{1}{V_{D}}-n \bar{I}_{11}\right]\right\} .
$$

Finally

$$
F_{4,1}=F_{5,1}=0
$$

\subsection{Summary}

Summarizing the previous results, the expansion of the susceptibility with DR is given by

$$
\chi=\frac{2}{n g_{0}^{2}}\left(1+g_{0}^{2} R_{1}+g_{0}^{4} R_{2}+\ldots\right)
$$

with

$$
R_{1}=-2(n-2) \bar{I}_{21}
$$

and

$$
R_{2}=R_{2}^{(a)}+R_{2}^{(b)}
$$

with

$$
\begin{aligned}
& R_{2}^{(a)}=2(n-2)\left\{-\bar{W}+2 \bar{I}_{21}\left[\bar{I}_{10}-\bar{I}_{21}\right]+\frac{2(n-2)}{V_{D}} \bar{I}_{31}\right\}, \\
& R_{2}^{(b)}=-4\left\{\frac{g_{4}^{(2)}}{4}\left[\frac{n_{1}}{V_{D}}-2 \bar{I}_{11}\right]+\frac{g_{4}^{(3)}}{4}\left[\frac{1}{V_{D}}-n \bar{I}_{11}\right]\right\} .
\end{aligned}
$$

\subsection{Case $n=2$}

Note that $R_{1}=0=R_{2}^{(a)}$ for $n=2$. This is easily seen since for this special case the 2-derivative action with chemical potential is simply

$$
A=\frac{1}{2 g_{0}^{2}} \int_{x}\left(\partial_{\mu} \Phi(x)-i h \delta_{\mu 0}\right)^{2}=\frac{1}{2 g_{0}^{2}} \int_{x}\left(\partial_{\mu} \Phi(x)\right)^{2}-\frac{h^{2}}{2 g_{0}^{2}} V_{D} .
$$

Therefore there are no corrections to the leading term for the susceptibility

$$
\chi=\frac{1}{g_{0}^{2}} .
$$




\subsection{Case $d=2$}

For $d=2$ the theory is renormalizable and as before we set the 4-derivative couplings to zero. Renormalization in the minimal subtraction (MS) scheme is achieved by

$$
g_{0}^{2}=\mu^{2 \epsilon} g_{\mathrm{MS}}^{2} Z_{1}
$$

with

$$
Z_{1}^{-1}=1+\frac{b_{0}}{2 \epsilon} g^{2}+\frac{b_{1}}{4 \epsilon} g^{4}+\ldots,
$$

where $b_{0}, b_{1}$ are as in (2.96).

For $D \sim 2$

$$
\bar{I}_{21} \sim-\frac{1}{4 \pi} L^{-D+2}\left[\frac{1}{D-2}-\frac{1}{2} \gamma_{2}+\kappa_{21}(D-2)+\ldots\right],
$$

where the functions $\gamma_{i}(\ell)$ are defined in [9].

Next

$$
\begin{aligned}
& \bar{I}_{10}=-\frac{1}{2 \pi} L^{-D+2}\left[\frac{1}{D-2}-\frac{1}{2} \alpha_{1}+\frac{1}{2 \mathcal{V}}+\kappa_{10}(D-2)+\ldots\right], \\
& \bar{I}_{31}=\frac{L^{2}}{64 \pi^{2}}\left[\gamma_{3}+1\right], \\
& \bar{W}=L^{-2 D+4} \frac{1}{8 \pi^{2}}\left[\frac{1}{(D-2)^{2}}+\frac{1}{(D-2)}\left(-\alpha_{1}-\frac{1}{2}+\frac{1}{\ell}\right)+\bar{w}+\ldots\right] .
\end{aligned}
$$

where $\alpha_{i}(\ell)$ are defined in [16].

In terms of the renormalized coupling

$$
\chi=\frac{2}{n g_{\mathrm{MS}}^{2}}\left\{1-b_{0}\left(\ln \left(\mu L_{s}\right)+\frac{1}{2} \gamma_{2}\right) g_{\mathrm{MS}}^{2}-b_{1}\left(\ln \left(\mu L_{s}\right)+r_{2}\right) g_{\mathrm{MS}}^{4}+\ldots\right\}
$$

with

$$
r_{2}=\bar{w}-2 \kappa_{10}-\frac{1}{2} \gamma_{2}\left(\alpha_{1}-\frac{1}{\ell}-\frac{1}{2} \gamma_{2}\right)-16 \pi^{2} \frac{(n-2)}{V_{D}} \bar{I}_{31} .
$$

For completeness we note that the free energy for large $h$ was computed to NLO with DR at infinite volume in $[17,18]$ with the result in the $\overline{\mathrm{MS}}$ scheme:

$$
\begin{aligned}
f(h)-f(0) & =-\frac{h^{2}}{2}\left[\frac{1}{g_{\overline{\mathrm{MS}}}^{2}(\mu)}-\frac{(n-2)}{2 \pi}\left(\ln (\mu / h)+\frac{1}{2}\right)+\mathrm{O}\left(g^{2}\right)\right] \\
& =-\frac{h^{2}}{2}\left[\frac{1}{g_{\overline{\mathrm{MS}}}^{2}(h)}-\frac{(n-2)}{4 \pi}+\mathrm{O}\left(g^{2}\right)\right] .
\end{aligned}
$$

Noting

$$
\frac{1}{g_{\overline{\mathrm{MS}}}^{2}(h)}=\frac{(n-2)}{2 \pi}\left[\ln \left(h / \Lambda_{\overline{\mathrm{MS}}}\right)+\frac{1}{n-2} \ln \ln \left(h / \Lambda_{\overline{\mathrm{MS}}}\right)+\ldots\right],
$$

this result can be expressed as

$$
f(h)-f(0)=-\frac{(n-2)}{2 \pi} \frac{h^{2}}{2}\left[\ln \frac{h}{\Lambda_{\overline{\mathrm{MS}}} \sqrt{\mathrm{e}}}+\frac{1}{(n-2)} \ln \ln \left(h / \Lambda_{\overline{\mathrm{MS}}}\right)+\ldots\right] .
$$


Eq. (3.71) was compared to the result from a non-perturbative computation invoking the Bethe ansatz $[17,18]$ thereby obtaining the exact ratio of the mass gap to the $\Lambda$-parameter $m / \Lambda_{\overline{\mathrm{MS}}}$. Later the thermodynamic Bethe ansatz equations were extended to study the spectrum at finite volume [19]-[22].

\subsection{Case $d=3$}

For $d=3$ the contribution of the 4-derivative terms and are not relevant at $\mathrm{O}\left(L_{s}^{-2}\right)$ since $R_{2}^{(b)}=\mathrm{O}\left(L_{s}^{-3}\right) .{ }^{8}$ We remark however that because the theory is non-renormalizable, it is expected that they are necessary to absorb divergences at higher orders.

For the sums contributing to $R_{1}, R^{(2 a)}$ we have

$$
\begin{aligned}
\bar{I}_{10} & =-\beta_{1} L_{s}^{-1}, \\
\bar{I}_{21} & =\frac{1}{8 \pi L_{s}}\left(\gamma_{2}-2\right), \\
& =-\frac{1}{3 L_{s}} \beta_{1} \text { for } \ell_{1}=\ell_{2}=\ell_{3}, \\
\bar{I}_{31} & =\frac{L_{s}}{64 \pi^{2}}\left(\gamma_{3}+2\right),
\end{aligned}
$$

where the functions $\beta_{i}(\ell), \gamma_{i}(\ell)$ are defined in [9]. Also $\bar{W}$ has a finite limit for $D=3$, and the results of numerical evaluation for $\ell=1, \ell=2$ are given in [9].

The agreement of $R_{1}, R_{2}$ with the lattice results is evident for $d=3$ because of the direct relation of the DR sums to the associated coefficients of the lattice sums:

$$
\bar{I}_{10}=I_{10 ; 1} / L_{s}, \quad \bar{I}_{21}=I_{21 ; 1} L_{s}, \quad \bar{I}_{31}=I_{31 ;-1} L_{s}, \quad \bar{W}=W_{3 a ; 2} / L_{s}^{2} .
$$

\subsection{Case $d=4$}

In $d=4$ we set $g_{0}^{2}=1 / F^{2}$ which is not renormalized with DR.

In ref. [9] we have computed the various functions appearing in $R_{1}, R_{2}$. First, for $d=4, \bar{I}_{21}$ has a finite limit as $q \rightarrow 0$ :

$$
\bar{I}_{21}=\frac{1}{8 \pi L_{s}^{2}}\left(\gamma_{2}(\ell)-1\right)+\mathrm{O}(D-4) .
$$

For $D \sim 4$ we find for the 1-loop functions,

$$
\begin{aligned}
& \bar{I}_{10}=-\beta_{1}(\ell) L_{s}^{-2}+\mathrm{O}(D-4), \\
& \bar{I}_{31}=\frac{1}{32 \pi^{2}}\left[\ln L_{s}-\frac{1}{D-4}+\frac{1}{2} \gamma_{3}(\ell)\right]+\mathrm{O}(D-4),
\end{aligned}
$$

and for the 2-loop function

$$
\bar{W}=\frac{1}{16 \pi^{2} L_{s}^{4}}\left\{\left[\frac{1}{D-4}-2 \ln L_{s}\right] \mathcal{W}_{0}(\ell)+\frac{1}{3 \ell} \ln (\hat{\ell})-\frac{10}{3} \mathcal{W}_{1}(\ell, \hat{\ell})+\overline{\mathcal{W}}(\ell)\right\}+\mathrm{O}(D-4),
$$

\footnotetext{
${ }^{8}$ Note that for $d=3$ the couplings $g_{4}^{(i)}$ have dimension, in contrast to $d=4$.
} 
with

$$
\mathcal{W}_{0}(\ell)=\frac{5}{3}\left(\frac{1}{2}-\gamma_{1}(\ell)\right)-\frac{1}{3 \ell} .
$$

$\overline{\mathcal{W}}(\ell)$ is given in section 4 of [9] (where $\bar{W}=-L^{4-2 D} \Psi$ ); also $\mathcal{W}_{1}(\ell, \hat{\ell})$ is given there albeit that explicit expression is not needed here.

Putting the results together for $D \sim 4$

$$
\begin{aligned}
R_{2}^{(a)}= & 2(n-2) \frac{1}{16 \pi^{2} L_{s}^{4}}\left\{-\left[\frac{1}{D-4}-2 \ln L_{s}\right]\left[\frac{5}{3}\left(\frac{1}{2}-\gamma_{1}(\ell)\right)+\frac{1}{\ell}\left(n-\frac{7}{3}\right)\right]\right. \\
& +\frac{1}{\ell}\left(n-\frac{7}{3}\right) \ln (\hat{\ell})+\frac{10}{3} \mathcal{W}_{1}(\ell, \hat{\ell}) \\
& \left.-\frac{1}{2}\left(\gamma_{2}(\ell)-1\right)^{2}-4 \pi \beta_{1}\left(\gamma_{2}(\ell)-1\right)+\frac{1}{2 \ell}(n-2) \gamma_{3}(\ell)-\overline{\mathcal{W}}(\ell)\right\} .
\end{aligned}
$$

For the 4-derivative terms we should identify

$$
\frac{g_{4}^{(2)}}{4}=-l_{1}, \quad \frac{g_{4}^{(3)}}{4}=-l_{2},
$$

with the bare couplings $l_{i}$ of Gasser and Leutwyler [2] for the standard $\overline{M S}$ scheme:

$$
l_{i}=\frac{w_{i}}{16 \pi^{2}}\left[\frac{1}{D-4}+\ln \left(\bar{c} \Lambda_{i}\right)\right]
$$

where $\ln \bar{c}=\bar{C}($ defined in $(4.3))$, and

$$
w_{1}=\frac{n}{2}-\frac{5}{3}, \quad w_{2}=\frac{2}{3},
$$

are as given by [2] in (2.141), (2.142). ${ }^{9}$ In order to pick up all terms of $R_{2}^{(b)}$ finite in the limit $D \rightarrow 4$ we need also order $q=D-4$ terms of $\bar{I}_{11}$ :

$$
\bar{I}_{11}=\frac{1}{L_{s}^{4}}\left\{\frac{1}{2}\left(1-q \ln L_{s}\right)\left[\gamma_{1}(\ell)-\frac{1}{2}\right]+q \mathcal{W}_{1}(\ell, \hat{\ell})\right\}+\mathrm{O}\left(q^{2}\right) .
$$

We then get for $D \sim 4$ :

$$
\begin{aligned}
R_{2}^{(b)}= & \frac{1}{16 \pi^{2} L_{s}^{4}}\left\{2(n-2)\left[\frac{1}{D-4}-2 \ln L_{s}\right]\left[\frac{5}{3}\left(\frac{1}{2}-\gamma_{1}(\ell)\right)+\frac{1}{\ell}\left(n-\frac{7}{3}\right)\right]\right. \\
& -2(n-2)\left[\frac{1}{\ell}\left(n-\frac{7}{3}\right) \ln (\hat{\ell})+\frac{10}{3} \mathcal{W}_{1}(\ell, \hat{\ell})\right] \\
& \left.+4 w_{1} \ln \left(\bar{c} \Lambda_{1} L_{s}\right)\left[\frac{(n-1)}{\ell}-\gamma_{1}(\ell)+\frac{1}{2}\right]+4 w_{2} \ln \left(\bar{c} \Lambda_{2} L_{s}\right)\left[\frac{1}{\ell}-\frac{n}{2}\left(\gamma_{1}(\ell)-\frac{1}{2}\right)\right]\right\} .
\end{aligned}
$$

Summing the terms we have for $d=4$ :

$$
\begin{aligned}
R_{2}= & \frac{1}{16 \pi^{2} L_{s}^{4}}\left\{-2(n-2)\left[\frac{1}{2}\left(\gamma_{2}-1\right)^{2}+4 \pi \beta_{1}\left(\gamma_{2}-1\right)-\frac{1}{2 \ell}(n-2) \gamma_{3}+\overline{\mathcal{W}}(\ell)\right]\right. \\
& \left.+4 w_{1} \ln \left(\bar{c} \Lambda_{1} L_{s}\right)\left[\frac{(n-1)}{\ell}-\gamma_{1}+\frac{1}{2}\right]+4 w_{2} \ln \left(\bar{c} \Lambda_{2} L_{s}\right)\left[\frac{1}{\ell}-\frac{n}{2}\left(\gamma_{1}-\frac{1}{2}\right)\right]\right\} .
\end{aligned}
$$

Here $\beta_{1}$ and $\gamma_{i}$ depend on $\ell=L_{0} / L_{s}$.

\footnotetext{
${ }^{9}$ In [2] only the $n=4$ result is given. Often the notation $\gamma_{i}$ is used for $w_{i}$ above, but we have already used $\gamma_{i}$ in the context of 1-loop integrals.
} 
Note that not only do the poles at $D=4$ cancel, but also $\mathcal{W}_{1}(\ell, \hat{\ell})$, hence the physical amplitude $R_{2}$ is independent of $\hat{\ell}$, the aspect ratio of the extra unphysical dimensions, as to be expected.

\section{Matching the effective actions for $d=2$ and $d=4$}

\subsection{Case $d=2$}

By matching the results for the susceptibility computed using lattice and dimensional regularizations we should obtain the 2-loop relation between the respective renormalized couplings

$$
g_{\text {latt }}^{2}=g_{\mathrm{MS}}^{2}\left[1+X_{1} g_{\mathrm{MS}}^{2}+X_{2} g_{\mathrm{MS}}^{4}+\ldots\right]
$$

First noting

$$
I_{21 ; 0}(\ell)=\frac{1}{8 \pi}\left[\gamma_{2}+2 \bar{C}+5 \ln 2\right]
$$

where

$$
\bar{C}=-\frac{1}{2}\left[\ln (4 \pi)-\gamma_{E}+1\right]=-1.476904292,
$$

at leading order we reproduce Parisi's result ${ }^{10}[23]$

$$
\begin{aligned}
X_{1} & =\bar{r}_{1}+\frac{1}{2} b_{0} \gamma_{2} \\
& =\frac{b_{0}}{2}\left[\ln \left(\frac{\pi}{8}\right)-\gamma_{E}\right]-\frac{1}{4} .
\end{aligned}
$$

The ratio of $\Lambda$ parameters is

$$
\frac{\Lambda_{\mathrm{latt}}}{\Lambda_{\mathrm{MS}}}=\exp \left(\frac{X_{1}}{b_{0}}\right)
$$

At next order matching we get

$$
X_{2}-X_{1}^{2}=\bar{r}_{2}+b_{1} r_{2} .
$$

For our purposes it is sufficient to consider the case $\ell=\hat{\ell}=1$ for which

$$
\bar{W}=\frac{1}{D}\left[\bar{I}_{10}^{2}-\frac{1}{V_{D}} \bar{I}_{20}\right], \quad(\ell=\hat{\ell}=1),
$$

so that

$$
\bar{w}=2 \kappa_{10}+\frac{1}{4} \alpha_{1}^{2}-\frac{1}{8}\left[\gamma_{3}+1\right], \quad(\ell=\hat{\ell}=1),
$$

and from (3.68) (noting $\gamma_{s}=(2 / d)(s-1) \alpha_{s-1}$ for $\ell=1$ )

$$
\begin{aligned}
r_{2} & =\frac{1}{2} \alpha_{1}-\frac{1}{4} \alpha_{2}-\frac{1}{8}-16 \pi^{2} \frac{(n-2)}{V_{D}} \bar{I}_{31} \\
& =-0.1022210828989128367197392-16 \pi^{2} \frac{(n-2)}{V_{D}} \bar{I}_{31}, \quad \ell=1
\end{aligned}
$$

\footnotetext{
${ }^{10}$ Converted from Pauli Villars regularization to DR.
} 
On the lattice side we get for $\ell=1$ from (2.111)

$$
\bar{r}_{2}=-\frac{5}{96}-(n-2)\left(\frac{1}{2 \pi} I_{10 ; 0}-I_{20 ;-2}-\frac{1}{32}+\frac{1}{16 \pi^{2}}+a_{1}\right)+4(n-2)^{2} I_{31 ;-2}
$$

where we used

$$
W_{3 a ; 0}=\frac{1}{2} I_{10 ; 0}^{2}-\frac{1}{2} I_{10 ; 0} I_{22 ; 0}-\frac{1}{2} I_{20 ;-2}+\frac{1}{2} a_{1}, \quad \ell=1
$$

and $a_{1}$ is the infinite-volume quantity

$$
\begin{aligned}
a_{1} & =-\frac{1}{4} \int_{k, l} \frac{E_{k+l}-E_{k}-E_{l}}{E_{k} E_{l} E_{k+l}^{2}} \sum_{\mu}{\widehat{(k+l})_{\mu}^{4}}^{4} \\
& =-\frac{1}{2} \sum_{x}(G(x)-G(0))^{2} \square_{0}^{2} G(x)=0.0461636292439177762(1)
\end{aligned}
$$

(with $E_{k}=\hat{k}^{2}$ ). Inserting the numerical values one gets

$$
\bar{r}_{2}=-\frac{5}{96}-(n-2) 0.02514054820286075900(1)+4(n-2)^{2} I_{31 ;-2}, \quad \ell=1 .
$$

Noting

$$
I_{31 ;-2}(\ell)=\frac{1}{L_{s}^{2} \ell} \bar{I}_{31}
$$

we obtain

$$
X_{2}-X_{1}^{2}=-\frac{5}{96}-1.0947301436539277 b_{1}
$$

$X_{2}$ was first computed by Falcioni and Treves [24]:

$$
X_{2}-X_{1}^{2}=-\frac{5}{96}+b_{1}\left[h_{1}-\frac{1}{4}+\frac{1}{2} \ln \left(\frac{\pi}{8}\right)-\frac{1}{2} \gamma_{E}\right]
$$

with the value of $h_{1}$ given in $[8]^{11}$

$$
h_{1}=-0.088766484(1)
$$

giving

$$
X_{2}-X_{1}^{2}=-\frac{5}{96}-1.094730144(1) b_{1} .
$$

The perfect agreement of our result (4.15) with the result obtained above by an independent method gives an additional check on our formulae in subsections 2.3.3), (3.4 which are valid for arbitrary $d \geq 2$.

\footnotetext{
${ }^{11} h_{1}=1 / 2-4 \pi^{2}\left(a_{1}-1 / 32\right)$ with $a_{1}$ given in (4.12). The value of $h_{1}$ given in [24] was not very precise.
} 


\subsection{Case $d=4$}

The equality of the lattice and DR results for $d=4$ at sub-leading order one requires

$$
I_{21 ; 2}=\frac{1}{8 \pi}\left(\gamma_{2}-1\right),
$$

which we have proven in [9].

Comparing (2.87)-(2.89) with (3.88), the coefficients of $\ln \left(L_{s}\right)$ agree due to the relation (see $[9])$ :

$$
I_{11 ; 4}=\frac{1}{2}\left(\gamma_{1}-\frac{1}{2}\right)
$$

For general $n$ the matching equation has the form

$$
H_{2} \bar{g}_{4,0}^{(2)}+H_{3} \bar{g}_{4,0}^{(3)}+H_{4} g_{4,0}^{(4)}+H_{5} g_{4,0}^{(5)}+H_{0}=0,
$$

where

$$
\begin{aligned}
& \bar{g}_{4,0}^{(2)}=g_{4,0}^{(2)}+\frac{1}{4 \pi^{2}} w_{1} \ln \left(a \bar{c} \Lambda_{1}\right)=-\frac{1}{4 \pi^{2}} w_{1} \ln \left(\frac{M_{2}}{\bar{c} \Lambda_{1}}\right), \\
& \bar{g}_{4,0}^{(3)}=g_{4,0}^{(3)}+\frac{1}{4 \pi^{2}} w_{2} \ln \left(a \bar{c} \Lambda_{2}\right)=-\frac{1}{4 \pi^{2}} w_{2} \ln \left(\frac{M_{3}}{\bar{c} \Lambda_{2}}\right),
\end{aligned}
$$

and

$$
H_{0}=\widehat{H}_{0}+2(n-2)\left[\frac{1}{16 \pi^{2}} \overline{\mathcal{W}}-2\left(I_{10 ; 2}-I_{21 ; 2}\right) I_{21 ; 2}\right]-\frac{(n-2)^{2}}{16 \pi^{2} \ell} \gamma_{3},
$$

where we have used another identity:

$$
I_{10 ; 2}=-\beta_{1} .
$$

So we have

$$
H_{0}=-(n-2) w+w^{\prime} I_{11 ; 4}+w^{\prime \prime} \frac{1}{\ell},
$$

with

$$
\begin{aligned}
w & =2 W_{3 a ; 4}-\frac{1}{8 \pi^{2}} \overline{\mathcal{W}}-\left(2 I_{10 ; 0}-\frac{1}{4}\right) I_{10 ; 4} \\
w^{\prime} & =\widehat{w}^{\prime} \\
w^{\prime \prime} & =\frac{1}{24}\left(3 n^{2}+n-12\right) I_{10 ; 0}-(n-2)\left\{\left(n-\frac{4}{3}\right) I_{32 ; 0}-\frac{1}{6} I_{33 ; 0}+\frac{1}{24}\right\}+4(n-2)^{2} i_{31 ; 0},
\end{aligned}
$$

where $\widehat{w}^{\prime}$ is given in (2.148) and $i_{31 ; 0}$ is defined by

$$
i_{31 ; 0}=I_{31 ; 0}(\ell)-\frac{1}{64 \pi^{2}} \gamma_{3}(\ell) .
$$

Now we find numerically

$$
i_{31 ; 0}=0.00211856418663447748445, \text { independent of } \ell,
$$

so that $w^{\prime \prime}$ is independent of $\ell$ (as is also $w^{\prime}$ ). 
Now the coefficients $H_{2}, H_{3}, H_{4}, H_{5}$ in (4.21) only involve the three linearly independent $\ell$-dependent functions $I_{00 ; 4}=-1 / \ell, I_{11 ; 4}$ and $J_{31 ; 4}-2 I_{22 ; 4}$ so that for consistency a relation for $w$ in (4.27) of the form

$$
w=\frac{d_{1}}{\ell}+d_{2} I_{11 ; 4}+d_{3}\left(J_{31 ; 4}-2 I_{22 ; 4}\right)
$$

should hold with some $\ell$-independent constants $d_{1}, d_{2}, d_{3}$. From numerical data sets with $\ell=1,2,3$ one finds $d_{1}=-0.00472740, d_{2}=0.00026214$ and $d_{3}=0.00000028$. Inserting these values into the relation with $\ell=4$ we indeed find consistency within the numerical errors (with the difference in the 6th significant digit). We will assume that actually $d_{3}=0$, and with this one obtains from $\ell=1,2$ the values $d_{1}=-0.00472752, d_{2}=0.00026215$.

Let us define

$$
\begin{aligned}
G_{1} & \equiv-n_{1} \bar{g}_{4,0}^{(2)}-\bar{g}_{4,0}^{(3)}+\frac{1}{6}\left(n_{1}+2\right) g_{4,0}^{(4)}+\frac{1}{2} n_{1} g_{4,0}^{(5)}, \\
G_{2} & \equiv 2 \bar{g}_{4,0}^{(2)}+\left(n_{1}+1\right) \bar{g}_{4,0}^{(3)}+\frac{2}{3}\left(n_{1}+2\right) g_{4,0}^{(4)}+\left(3 n_{1}-1\right) g_{4,0}^{(5)} .
\end{aligned}
$$

Then matching requires

$$
\begin{aligned}
& 0=G_{1}+q_{0}^{(1)}+(n-2) q_{1}^{(1)}+(n-2)^{2} q_{2}^{(1)}, \\
& 0=G_{2}+q_{0}^{(2)}+(n-2) q_{1}^{(2)} \\
& 0=2 g_{4,0}^{(5)}-d_{3}
\end{aligned}
$$

with

$$
\begin{aligned}
& q_{0}^{(1)}=\frac{1}{12} I_{10 ; 0}, \\
& q_{1}^{(1)}=-\frac{1}{24}+\frac{13}{24} I_{10 ; 0}-\frac{2}{3} I_{32 ; 0}+\frac{1}{6} I_{33 ; 0}-d_{1}, \\
& q_{2}^{(1)}=\frac{1}{8} I_{10 ; 0}-I_{32 ; 0}+4 i_{31 ; 0}, \\
& q_{0}^{(2)}=-\frac{3}{16}+\frac{5}{6} I_{10 ; 0}, \\
& q_{1}^{(2)}=-\frac{5}{48}+\frac{2}{3} I_{10 ; 0}-\frac{8}{3} I_{32 ; 0}+\frac{2}{3} I_{33 ; 0}-d_{2} .
\end{aligned}
$$

The numerical values are

$$
\begin{aligned}
& q_{0}^{(1)}=0.0129111158, \\
& q_{1}^{(1)}=0.0434608716, \\
& q_{2}^{(1)}=0.011640543735, \\
& q_{0}^{(2)}=-0.0583888414, \\
& q_{1}^{(2)}=-0.0152288420 .
\end{aligned}
$$

For the special case $n=2$ the solution is:

$$
\begin{array}{ll}
g_{4,0}^{(4)}+g_{4,0}^{(5)}=\frac{1}{16}-\frac{1}{3} I_{10 ; 0}, & (n=2), \\
\bar{g}_{4,0}^{(2)}+\bar{g}_{4,0}^{(3)}=\frac{1}{32}-\frac{1}{12} I_{10 ; 0}, & (n=2) .
\end{array}
$$

Note in the continuum limit (e.g. for DR) $A_{4}^{(2)}=A_{4}^{(3)}$ for $n=2$. 


\section{The mass gap}

The mass of the $\mathrm{O}(n)$ vector particle in a periodic spatial volume $L_{s}^{d-1}$ was computed with lattice regularization for arbitrary $d$ in ref. [7] up to second order in perturbation theory. It takes the form

$$
m_{1}=\frac{n_{1} g_{0}^{2} a^{d-2}}{2 L_{s}^{d-1}}\left\{1+g_{0}^{2} c_{2}\left(a / L_{s}\right)+g_{0}^{4}\left[c_{3}\left(a / L_{s}\right)+\sum_{j=2}^{5} g_{4,0}^{(j)} d_{3}^{(j)}\left(a / L_{s}\right)\right]+\mathrm{O}\left(g_{0}^{6}\right)\right\},
$$

where the coefficients $c_{2}\left(a / L_{s}\right), c_{3}\left(a / L_{s}\right), d_{3}\left(a / L_{s}\right),{ }^{12}$ are given in appendix B of [7]; they depend on $d$, and for the case $d=2$ the coefficients $c_{2}, c_{3}$ agree with those previously computed in [8].

Here we will only discuss the case $d=4$. Results are often quoted in terms of the moment of inertia $\Theta$ which is simply related to the mass gap through

$$
m_{1}=\frac{(n-1)}{2 \Theta} .
$$

$\Theta$ has a perturbative expansion of the form

$$
\frac{\Theta}{F^{2} L_{s}^{3}}=1+\Theta_{1}\left(F L_{s}\right)^{-2}+\Theta_{2}\left(F L_{s}\right)^{-4}+\ldots
$$

After renormalization of the couplings as in subsection 2.4.3, the moment of inertia in the continuum limit is given by (5.3) with coefficients determined from the lattice computation taken from eq. (6.20) of [7]

$$
\Theta_{1}^{\text {latt }}=0.225784959441(n-2),
$$

and

$$
\begin{aligned}
\Theta_{2}^{\text {latt }}= & -\frac{0.8375369106}{12 \pi^{2}}\left[(3 n-10) \ln \left(M_{2} L_{s}\right)+2 n \ln \left(M_{3} L_{s}\right)\right] \\
& +0.55835794046(n+1) g_{4,0}^{(4)} \\
& +(1.11639602502 n-0.55771822866) g_{4,0}^{(5)} \\
& -0.0489028095+0.0101978424(n-2) .
\end{aligned}
$$

Using the definitions in (4.22), (4.23) and (4.34) we can rewrite this involving DR scales:

$$
\Theta_{2}^{\text {latt }}=\bar{\theta}_{2}-\frac{0.8375369106}{12 \pi^{2}}\left[(3 n-10) \ln \left(\bar{c} \Lambda_{1} L_{s}\right)+2 n \ln \left(\bar{c} \Lambda_{2} L_{s}\right)\right],
$$

with

$$
\begin{aligned}
\bar{\theta}_{2}= & 0.8375369106 G_{2}-1.396214707(n-2) g_{4,0}^{(5)} \\
& -0.0489028095+0.0101978424(n-2) .
\end{aligned}
$$

\footnotetext{
${ }^{12}$ Keeping the notation of [7] and not to be confused with previously mentioned quantities with the same letters!
} 
Finally using eq. (4.36) (with (4.46), (4.47)) which was obtained by matching lattice and DR results for the free energy, and assuming $g_{4,0}^{(5)}=0$, we obtain

$$
\bar{\theta}_{2}=0.0229525597(n-2) \text {. }
$$

Note that the $(n-2)^{0}$ terms in $\bar{\theta}_{2}$ cancel to our numerical precision of 10 digits.

The continuum limit of $\Theta_{i}$ should of course be regularization independent. Unfortunately (5.8) does not agree with the result for the moment of inertia previously computed by Hasenfratz [5] using dimensional regularization. For this reason we recomputed the mass gap with DR using free boundary conditions in the time direction in an analogous way to that used for the lattice computation. The computation is rather lengthy and here we only present the final result (for arbitrary $d$ ):

$$
m_{1}=\frac{n_{1} g_{0}^{2}}{2 \bar{V}_{D}}\left[1+g_{0}^{2} \triangle^{(2)}+g_{0}^{4} \triangle^{(3)}+\ldots\right]
$$

(here $\left.\bar{V}_{D}=L_{s}^{d-1} \widehat{L}^{q}=L_{s}^{D-1} \hat{\ell}^{q}\right)$, with

$$
\begin{aligned}
& \triangle^{(2)}=(n-2) R(0) \\
& \triangle^{(3)}=(n-2)\left[2 W+\frac{3}{4 \bar{V}_{D}} \bar{I}_{10: D-1}+(n-3) R(0)^{2}\right]-4\left(2 l_{1}+n l_{2}\right) \ddot{R}(0) .
\end{aligned}
$$

Here $R(z)$ is the propagator for an infinitely long strip without the slow modes $\mathbf{p}=0:{ }^{13}$

$$
R(z)=\frac{1}{2 \bar{V}_{D}} \sum_{\mathbf{p} \neq 0} \frac{\mathrm{e}^{-\omega(\mathbf{p})\left|z_{0}\right|+i \mathbf{p z}}}{\omega(\mathbf{p})}, \omega(\mathbf{p})=\sqrt{\mathbf{p}^{2}} .
$$

The singularity of $R(z)$ at $z=0$ is regularized with DR. Further $\bar{I}_{10: D-1}$ is the regularized sum $\bar{I}_{10}$ in $D-1$ dimensions and

$$
W=-\int_{-\infty}^{\infty} \mathrm{d} z_{0} \int_{\mathbf{z}} R(z)^{2} \partial_{0}^{2} R(z) .
$$

The computation of $W$ is the most involved part and we discuss this in detail in section 5 of [9] (where $W=-L^{4-2 D} \bar{\Psi}$ ).

Returning again to the case $d=4$, the moment of inertia has an expansion

$$
\frac{\Theta}{F^{2} L_{s}^{3}}=1+\Theta_{1}^{\mathrm{DR}}\left(F L_{s}\right)^{-2}+\Theta_{2}^{\mathrm{DR}}\left(F L_{s}\right)^{-4}+\ldots
$$

with

$$
\begin{aligned}
& \Theta_{1}^{\mathrm{DR}}=-(n-2) L_{s}^{2} R(0) \\
& \Theta_{2}^{\mathrm{DR}}=(n-2) L_{s}^{4}\left[-2 W+R(0)^{2}-\frac{3}{4 \bar{V}_{D}^{2}} \sum_{\mathbf{p} \neq 0} \frac{1}{\mathbf{p}^{2}}\right]+4\left(2 l_{1}+n l_{2}\right) L_{s}^{4} \ddot{R}(0) .
\end{aligned}
$$

\footnotetext{
${ }^{13} \operatorname{Our} R(z)$ is closely related to $\bar{G}^{*}(z)$ of [5].
} 
In [9] we find

$$
W=\frac{5}{24 \pi^{2}} \ddot{R}(0)\left[\frac{1}{D-4}-\ln L_{s}\right]+c_{w} L_{s}^{-4}
$$

with $^{14}$

$$
c_{w}=0.0986829798 \text {. }
$$

Adding the counter-terms using (3.84), the 1/(D-4) singularities cancel (and also the $\hat{\ell}$-dependent terms coming from $\mathrm{O}(D-4)$ contributions in $\ddot{R}(0))$, and we obtain

$$
\Theta_{2}^{\mathrm{DR}}=(n-2) \theta_{2}+\frac{1}{12 \pi^{2}} L_{s}^{4} \ddot{R}(0)\left[(3 n-10) \ln \left(\bar{c} \Lambda_{1} L_{s}\right)+2 n \ln \left(\bar{c} \Lambda_{2} L_{s}\right)\right],
$$

with $^{15}$

$$
\theta_{2}=-2 c_{w}+L_{s}^{4} R(0)^{2}+\frac{3}{4} L_{s} \beta_{1}^{(3)}
$$

where in the notation of $[16]$

$$
\beta_{1}^{(3)}=-\frac{1}{\bar{V}_{D}} \sum_{\mathbf{p} \neq 0} \frac{1}{\mathbf{p}^{2}}
$$

where the sum is over $3+q$ dimensional momenta $\mathbf{p}$. With dimensional regularization

$$
\beta_{1}^{(3)}=-L_{s} R(0)
$$

Putting in the numerical values [9]

$$
\begin{aligned}
& L_{s}^{2} R(0)=-0.2257849594407580334832664917, \\
& L_{s}^{4} \ddot{R}(0)=-0.8375369106960818783868948293,
\end{aligned}
$$

we obtain

$$
\theta_{2}=0.0229516079
$$

completely consistent with the lattice result converted to DR in (5.8). Note however that values of $\theta_{2}, \bar{\theta}_{2}$ differ in the 6 'th decimal place, which indicates that at some stage(s) we have overestimated our numerical precision.

\section{Conclusions}

We have established relations between the 4-derivative couplings of effective Lagrangians involving fields in the vector representation of $\mathrm{O}(n)$ using both lattice and dimensional regularizations. This allows translation of results obtained on the lattice to those of DR more commonly used in phenomenology. Computations on the lattice are usually algebraically more complicated but conceptually clear.

\footnotetext{
${ }^{14}$ The value $c_{w}=0.029492025146$ given in [5] differs from ours.

${ }^{15}$ In ref. [5] the term in (5.20) involving $\beta_{1}^{(3)}$ is missing.
} 
One application is to the computation of the mass gap of massless 2-flavor QCD in the $\delta$-regime. It is given by

$$
\begin{aligned}
\Theta= & F^{2} L_{s}^{3}\left[1+0.4515699182 \frac{1}{F^{2} L_{s}^{2}}\right. \\
& \left.+\frac{1}{F^{4} L_{s}^{4}}\left(\theta-0.8375369109 \frac{1}{6 \pi^{2}}\left\{\ln \left(\Lambda_{1} L_{s}\right)+4 \ln \left(\Lambda_{2} L_{s}\right)\right\}\right)+\ldots\right],
\end{aligned}
$$

with

$$
\begin{aligned}
\theta & =2 \theta_{2}+\frac{5}{6 \pi^{2}} L_{s}^{4} \ddot{R}(0) \bar{C} \\
& =0.1503452489 .
\end{aligned}
$$

Note Hasenfratz [5] obtained $\theta=0.088431628$.

It is convenient to rewrite (6.1) by using the low-energy parameters defined in [2],

$$
\bar{l}_{i} \equiv \ln \frac{\Lambda_{i}^{2}}{m_{\pi}^{2}},
$$

where $m_{\pi}$ is the physical pion mass. We have

$$
\begin{aligned}
\Theta= & F^{2} L_{s}^{3}\left[1+\frac{0.45157}{F^{2} L_{s}^{2}}\right. \\
& \left.+\frac{1}{F^{4} L_{s}^{4}}\left(0.1503-0.0283\left[\bar{l}_{2}+\frac{1}{4} \bar{l}_{1}+\frac{5}{2} \ln \left(L_{s} m_{\pi}\right)\right]\right)+\ldots\right] .
\end{aligned}
$$

The QCDSF collaboration [25-27] compared their data for the mass gap from numerical simulations of lattice QCD to (6.1) using values

$$
\bar{l}_{1}=-0.4 \pm 0.6, \quad \bar{l}_{2}=4.3 \pm 0.1 .
$$

taken from [28]. They found satisfactory agreement with the analytic result and our new value for $\theta$ doesn't change this conclusion.

Although measuring the low lying spectrum is among the simplest and cleanest numerical problems, a difficulty is that the box size needs to reach $3 \mathrm{fm}$ or larger. This is suggested by the NLO correction which is $38 \%, 26 \%$ and $15 \%$ of the leading order for $L_{s}=2.5,3,4$ fermi respectively, where for the estimates we have used the value $F=86.2 \mathrm{MeV}$ from Colangelo and Dür [29]. The NNLO correction is unexpectedly small: $-0.6 \%,-0.7 \%$ and $-0.5 \%$ at the same lattice sizes. Note however, that this is due to the cancellation of the two terms in (6.4), and the smallness of the NNLO correction does not indicate the smallness of the next, unknown correction.

Note that the combination $\bar{l}_{2}+\bar{l}_{1} / 4$ enters with a small coefficient, whose value e.g. for $L_{s}=3 \mathrm{fm}$ is -0.0095 . As a consequence, the mass gap is not sensitive to these parameters. For the same reason, however, it provides a clean way to obtain the value of $F$, in particular the constant $\bar{l}_{4}$ which controls the ratio $F_{\pi} / F$ close to the chiral limit. At the physical pion mass the sensitivity of the mass gap in the delta regime to this parameter is roughly 


\begin{tabular}{|l|r|r|r|}
\hline$\ell$ & \multicolumn{1}{|c|}{$e_{1}$} & \multicolumn{1}{c|}{$d_{1}$} & \multicolumn{1}{c|}{$d_{2}$} \\
\hline 3 & -0.548430 & -0.000035 & -0.014214 \\
2 & -0.215019 & 0.003484 & -0.007174 \\
1 & 0.140461 & 0.014776 & 0.016887 \\
0.5 & 0.666497 & 0.069766 & 0.194629 \\
0.25 & 2.666667 & 0.761772 & 2.878218 \\
\hline
\end{tabular}

Table 1. The shape dependence of the coefficients in (6.7).

$0.2 \bar{l}_{4}$. Alternatively, knowing $F$, one can estimate the corresponding lattice artifacts, the goodness of the chiral extrapolation, etc.

Numerical simulations of lattice QCD in the $\delta$-regime potentially still give a good possibility to constrain the LE constants of $\chi P T$. The mass gap is unfortunately only sensitive to the decay constant $F$, and it remains a challenge to find other observables which are sensitive to the $\bar{l}_{i}$ and also accurately measurable in numerical simulations.

The susceptibility discussed in this paper could also serve as such an alternative quantity. To use this one has to consider massless Ginsparg-Wilson fermions (having exact chiral symmetry [30]) and using the associated conserved currents [31, 32]. This choice has the advantage that one has an extra parameter, $\ell$, which affects the sensitivity to the LEC's $\bar{l}_{1}$ and $\bar{l}_{2}$. Writing the susceptibility for $n=4$ as

$$
\chi=\frac{1}{2 F^{2}}\left[1+\frac{1}{F^{2} L^{2}} e_{1}+\frac{1}{F^{4} L^{4}}\left(e_{2}+d_{1} \bar{l}_{1}+d_{2} \bar{l}_{2}\right)+\mathrm{O}\left(\frac{1}{F^{6} L^{6}}\right)\right],
$$

one has (cf. (3.54), (3.55) and (3.88))

$$
\begin{aligned}
& e_{1}=-\frac{1}{2 \pi}\left(\gamma_{2}-1\right) \\
& d_{1}=-\frac{1}{24 \pi^{2}}\left(\gamma_{1}-\frac{3}{\ell}-\frac{1}{2}\right) \\
& d_{2}=-\frac{1}{6 \pi^{2}}\left(\gamma_{1}-\frac{1}{2 \ell}-\frac{1}{2}\right)
\end{aligned}
$$

The shape dependence of the coefficients $e_{1}, d_{1}, d_{2}$ is illustrated ${ }^{16}$ in table 1 . As one can see, the sensitivity to $\bar{l}_{i}$ increases with decreasing $\ell$. This can perhaps be understood: at $h>0$ for large $\ell$ (large time direction) the state with maximal $Q_{12}$ in an isospin multiplet is dominating, while decreasing $\ell$ all other states from the given multiplets start to influence the free energy, and because of nearly degenerate states this increases the susceptibility. Note, however, that the NLO coefficient $e_{1}(\ell)$ also increases (although not as fast as the NNLO coefficients) and at fixed size $L_{s}=L$ one cannot trust the expansion for too small values of $\ell$.

\footnotetext{
${ }^{16}$ We did not calculate the 2-loop contribution $\overline{\mathcal{W}}\left(\ell\right.$ ) (needed for $e_{2}$ ) for $\ell<1$.
} 


\section{Acknowledgments}

Christoph Weiermann participated at an early stage of these calculations. We thank him for the collaboration. We also would like to thank Janos Balog, Gilberto Colangelo, Peter Hasenfratz, Heiri Leutwyler and Martin Lüscher for useful discussions.

\section{A The terms $B_{4}^{(i)}$ and $C_{4}^{(i)}$}

The terms $B_{4}^{(i)}$ and $C_{4}^{(i)}$ appearing in (2.48) are given by

$$
\begin{aligned}
B_{4}^{(1)} & =B_{4}^{(5 a)}+B_{4}^{(1 a)}, \\
B_{4}^{(1 a)} & =4 \sum_{x k}\left(\square_{k} S_{1} \nabla_{0} S_{2}-\square_{k} S_{2} \nabla_{0} S_{1}\right), \\
C_{4}^{(1)} & =C_{4}^{(5 a)}+C_{4}^{(1 a)}, \\
C_{4}^{(1 a)} & =\sum_{x k}\left\{\left(2 S_{1}+\square_{0} S_{1}\right) \square_{k} S_{1}+\left(2 S_{2}+\square_{0} S_{2}\right) \square_{k} S_{2}\right\},
\end{aligned}
$$

where $\nabla_{0}=\frac{1}{2}\left(\partial_{0}+\partial_{0}^{*}\right)$ is the symmetric derivative and $\square_{\mu}=\partial_{\mu} \partial_{\mu}^{\star}$. Here and in the rest of this section we have suppressed the argument of the fields e.g. $S_{i}=S_{i}(x)$. Also below we introduce the notation $S_{i}^{\prime}=S_{i}(x+\hat{0})$ and $S_{i}^{\prime \prime}=S_{i}(x+2 \hat{0})$.

$$
\begin{aligned}
B_{4}^{(2)} & =-4 \sum_{x}\left(S_{1} S_{2}^{\prime}-S_{2} S_{1}^{\prime}\right) \partial_{\mu} \mathbf{S} \cdot \partial_{\mu} \mathbf{S} \\
C_{4}^{(2)} & =-\sum_{x}\left\{4\left(S_{1} S_{2}^{\prime}-S_{2} S_{1}^{\prime}\right)^{2}+2\left(S_{1} S_{1}^{\prime}+S_{2} S_{2}^{\prime}\right) \partial_{\mu} \mathbf{S} \cdot \partial_{\mu} \mathbf{S}\right\} \\
B_{4}^{(3)} & =B_{4}^{(4 a)}+B_{4}^{(3 a)} \\
B_{4}^{(3 a)} & =-4 \sum_{x k}\left(S_{1}^{\prime} \partial_{k} S_{2}-S_{2}^{\prime} \partial_{k} S_{1}\right) \partial_{0} \mathbf{S} \cdot \partial_{k} \mathbf{S} \\
C_{4}^{(3)} & =C_{4}^{(4 a)}+C_{4}^{(3 a)} \\
C_{4}^{(3 a)} & =2 \sum_{x k}\left[\left(S_{1}^{\prime} \partial_{k} S_{1}+S_{2}^{\prime} \partial_{k} S_{2}\right) \partial_{0} \mathbf{S} \cdot \partial_{k} \mathbf{S}-\left(S_{1}^{\prime} \partial_{k} S_{2}-S_{2}^{\prime} \partial_{k} S_{1}\right)^{2}\right] \\
B_{4}^{(4 a)} & =-4 \sum_{x}\left(S_{1} S_{2}^{\prime}-S_{2} S_{1}^{\prime}\right) \partial_{0} \mathbf{S} \cdot \partial_{0} \mathbf{S} \\
C_{4}^{(4 a)} & =-\sum_{x}\left\{2\left(S_{1} S_{1}^{\prime}+S_{2} S_{2}^{\prime}\right) \partial_{0} \mathbf{S} \cdot \partial_{0} \mathbf{S}+4\left(S_{1} S_{2}^{\prime}-S_{2} S_{1}^{\prime}\right)^{2}\right\} \\
B_{4}^{(5 a)} & =-4 \sum_{x}\left(\partial_{0}^{*} S_{1} \partial_{0} S_{2}-\partial_{0}^{*} S_{2} \partial_{0} S_{1}\right) \\
C_{4}^{(5 a)} & =-2 \sum_{x}\left\{-\left(S_{1} \square_{0} S_{1}+S_{2} \square_{0} S_{2}\right)+2\left(\partial_{0} S_{1} \partial_{0}^{*} S_{1}+\partial_{0} S_{2} \partial_{0}^{*} S_{2}\right)\right\}
\end{aligned}
$$




$$
\begin{aligned}
& B_{4}^{(5 c)}=B_{4}^{(5 a)}+B_{4}^{(5 d)}, \\
& B_{4}^{(5 d)}=-4 \sum_{x}\left\{\left[S_{1}^{\prime \prime}-S_{1}^{\prime}\right] \partial_{k}^{2} S_{2}-\left[S_{2}^{\prime \prime}-S_{2}^{\prime}\right] \partial_{k}^{2} S_{1}\right\} \\
& C_{4}^{(5 c)}=C_{4}^{(5 a)}+C_{4}^{(5 d)}, \\
& C_{4}^{(5 d)}=2 \sum_{x k}\left\{\left[2 S_{1}^{\prime \prime}-S_{1}^{\prime}\right] \partial_{k}^{2} S_{1}+\left[2 S_{2}^{\prime \prime}-S_{2}^{\prime}\right] \partial_{k}^{2} S_{2}\right\} .
\end{aligned}
$$

\section{B Some lattice momentum sums}

We define the following lattice sums:

$$
\begin{aligned}
I_{n m} & \equiv \frac{1}{V} \sum_{p}^{\prime} \frac{\left(\hat{p}_{0}^{2}\right)^{m}}{\left(\hat{p}^{2}\right)^{n}}, \\
J_{n m} & \equiv \frac{1}{V} \sum_{p}^{\prime} \frac{\left(\hat{p}_{0}^{2}\right)^{m} \sum_{\mu} \hat{p}_{\mu}^{4}}{\left(\hat{p}^{2}\right)^{n}}, \\
K_{n m} & \equiv \frac{1}{V} \sum_{p}^{\prime} \frac{\left(\hat{p}_{0}^{2}\right)^{m}\left(\sum_{\mu} \hat{p}_{\mu}^{4}\right)^{2}}{\left(\hat{p}^{2}\right)^{n}}, \\
L_{n m} & \equiv \frac{1}{V} \sum_{p}^{\prime} \frac{\left(\hat{p}_{0}^{2}\right)^{m}}{\left(\hat{p}^{2}\right)^{n} \sum_{\mu \nu} \cos \left(p_{\mu}-p_{\nu}\right) \hat{p}_{\mu}^{2} \hat{p}_{\nu}^{2},} \\
J_{n m k} & \equiv \frac{1}{V} \sum_{p}^{\prime} \frac{\left(\hat{p}_{0}^{2}\right)^{m} \sum_{\mu} \hat{p}_{\mu}^{2 k}}{\left(\hat{p}^{2}\right)^{n}} .
\end{aligned}
$$

The following momentum sums which appear in our computation are expressed in terms of these:

$$
\begin{aligned}
\mathcal{F}_{1} \equiv & \frac{1}{V^{2}} \sum_{p q}^{\prime} \frac{\hat{p}_{0}^{2}(\widehat{p+q})^{2}}{\left(\hat{p}^{2}\right)^{2} \hat{q}^{2}} \\
= & I_{11} I_{10}+I_{21} I_{00}-\frac{d_{s}+1}{2 d_{s}} I_{22} I_{11}-\frac{1}{2 d_{s}} I_{11} I_{00}+\frac{1}{2 d_{s}} I_{11}^{2}+\frac{1}{2 d_{s}} I_{22} I_{00} . \\
\mathcal{F}_{2} \equiv & \frac{1}{V^{2}} \sum_{p q}^{\prime} \frac{\sin ^{2} p_{0}(\widehat{p+q})^{2}}{\left(\hat{p}^{2}\right)^{3} \hat{q}^{2}} \\
= & I_{10}\left(I_{21}-\frac{1}{4} I_{22}\right)+I_{00}\left(I_{31}-\frac{1}{4} I_{32}\right)-\frac{1}{2} I_{11}\left(I_{32}-\frac{1}{4} I_{33}\right) \\
& +\frac{1}{2 d_{s}}\left(I_{11}-I_{00}\right)\left(I_{21}-\frac{1}{4} I_{22}-I_{32}+\frac{1}{4} I_{33}\right) .
\end{aligned}
$$

The expressions for $\mathcal{F}_{1}$ and $\mathcal{F}_{2}$ are valid for a spatially symmetric volume, $V=L_{s}^{d_{s}} L_{t}$.

$$
\begin{aligned}
\mathcal{F}_{3} & \equiv \frac{1}{V^{2}} \sum_{p q}^{\prime} \frac{\sin p_{0} \sin q_{0}(\widehat{p+q})^{2}}{\left(\hat{p}^{2}\right)^{2}\left(\hat{q}^{2}\right)^{2}} \\
& =2\left(\frac{1}{V} \sum_{p}^{\prime} \frac{\sin ^{2} p_{0}}{\left(\hat{p}^{2}\right)^{2}}\right)^{2}=2\left(I_{21}-\frac{1}{4} I_{22}\right)^{2} .
\end{aligned}
$$




$$
\begin{aligned}
\mathcal{F}_{4} & =L_{21}=\frac{1}{V} \sum_{p}^{\prime} \frac{\hat{p}_{0}^{2}}{\left(\hat{p}^{2}\right)^{2}} \sum_{\mu \nu} \cos \left(p_{\mu}-p_{\nu}\right) \hat{p}_{\mu}^{2} \hat{p}_{\nu}^{2} \\
& =I_{01}-J_{11}+\frac{1}{4} K_{21}+J_{213}-\frac{1}{4} J_{214} \cdot \\
\mathcal{F}_{5} & =\frac{1}{V} \sum_{p}^{\prime} \frac{\sin ^{2} p_{0}}{\left(\hat{p}^{2}\right)^{3}} \sum_{\mu \nu} \cos \left(p_{\mu}-p_{\nu}\right) \hat{p}_{\mu}^{2} \hat{p}_{\nu}^{2}=L_{31}-\frac{1}{4} L_{32} \\
& =I_{11}-J_{21}+\frac{1}{4} K_{31}+J_{313}-\frac{1}{4} J_{314}-\frac{1}{4}\left[I_{12}-J_{22}+\frac{1}{4} K_{32}+J_{323}-\frac{1}{4} J_{324}\right] . \\
\mathcal{F}_{6} & \equiv \frac{1}{V} \sum_{p}^{\prime} \frac{1}{\hat{p}^{2}} \sum_{k}\left[2 \cos \left(2 p_{0}\right)-\cos \left(p_{0}\right)\right] \cos \left(p_{k}\right) \hat{p}_{k}^{2} \\
& =I_{00}-\frac{7}{2} I_{01}+I_{02}-\frac{1}{2} J_{10}+\frac{7}{4} J_{11}-\frac{1}{2} J_{12}-I_{11}+4 I_{12}-\frac{11}{4} I_{13}+\frac{1}{2} I_{14} . \\
\mathcal{F}_{7} & \equiv \frac{1}{V} \sum_{p}^{\prime} \sum_{k} \frac{\left(\mathrm{e}^{-i p_{0}}-1\right)\left(\mathrm{e}^{-i 2 p_{0}}-1\right)\left(\mathrm{e}^{i p_{k}}-1\right)^{2}}{\left(\hat{p}^{2}\right)^{2}} \\
& =-\frac{1}{V} \sum_{p}^{\prime} \sum_{k} \frac{\hat{p}_{k}^{2} \cos p_{k}\left(\cos 3 p_{0}-\cos 2 p_{0}-\cos p_{0}+1\right)}{\left(\hat{p}^{2}\right)^{2}} \\
& =2 I_{11}-\frac{5}{2} I_{12}+\frac{1}{2} I_{13}-2 I_{22}+\frac{7}{2} I_{23}-\frac{7}{4} I_{24}+\frac{1}{4} I_{25}-J_{21}+\frac{5}{4} J_{22}-\frac{1}{4} J_{23} .
\end{aligned}
$$

\section{Correlators appearing in $\boldsymbol{F}_{r}$ with lattice regularization}

\section{C.1 Correlators appearing in $F_{1,0}$ and $F_{1,1}$}

First

$$
\left\langle U_{1}\right\rangle_{0}=n_{1} \frac{1}{V} \sum_{p}^{\prime} \frac{\hat{p}_{0}^{2}}{\hat{p}^{2}}=n_{1} I_{11},
$$

where $I_{n m}$ are defined in (B.1). Next

$$
\begin{aligned}
\left\langle U_{2}\right\rangle_{0} & =\left.\partial_{0}^{x} \partial_{0}^{y} \frac{1}{4 V} \sum_{x}\left\langle\vec{\pi}(x)^{2} \vec{\pi}(y)^{2}\right\rangle_{0}\right|_{y=x}=\left.\frac{n_{1}}{2} \partial_{0}^{x} \partial_{0}^{y}\left[\frac{1}{V} \sum_{x} G(x-y)^{2}\right]\right|_{y=x} \\
& =\frac{n_{1}}{V^{2}} \sum_{p q}^{\prime} \frac{1-\cos \left(p_{0}+q_{0}\right)}{\hat{p}^{2} \hat{q}^{2}}=n_{1} I_{11}\left(I_{10}-\frac{1}{4} I_{11}\right) \\
\left\langle U_{1} A_{2,0}\right\rangle_{0}^{c} & =n_{1} I_{11}, \\
\left\langle U_{1} A_{2,1}^{(a)}\right\rangle_{0}^{c} & =-n_{1}\left(1-\frac{n_{1}}{V}\right) I_{21} \\
\left\langle U_{1} A_{2,1}^{(b)}\right\rangle_{0}^{c} & =\left.\frac{1}{8 V} \sum_{x u} \partial_{\mu}^{u} \partial_{\mu}^{v}\left\langle\partial_{0} \vec{\pi}(x) \cdot \partial_{0} \vec{\pi}(x) \vec{\pi}(u)^{2} \vec{\pi}(v)^{2}\right\rangle_{0}^{c}\right|_{v=u} \\
& =\left.\frac{n_{1}}{V} \sum_{x u} \partial_{\mu}^{u} \partial_{\mu}^{v}\left\{\partial_{0}^{x} G(x-u) \partial_{0}^{x} G(x-v) G(u-v)\right\}\right|_{v=u} \\
& =n_{1} \mathcal{F}_{1}
\end{aligned}
$$


where $\mathcal{F}_{1}$ is given by (B.6). Also ${ }^{17}$

$$
\begin{aligned}
\left\langle U_{1} A_{4,1}^{(1)}\right\rangle_{0}^{c} & =2 n_{1} I_{01}, \\
\left\langle U_{1} A_{4,1}^{(i)}\right\rangle_{0}^{c} & =0, \quad i=2,3,4, \\
\left\langle U_{1} A_{4,1}^{(5 a)}\right\rangle_{0}^{c} & =2 n_{1} J_{21}, \\
\left\langle U_{1} A_{4,1}^{(5 b)}\right\rangle_{0}^{c} & =2 n_{1} I_{01}, \\
\left\langle U_{1} A_{4,1}^{(5 c)}\right\rangle_{0}^{c} & =2 n_{1} \sum_{x} \partial_{0}^{*} \partial_{\mu} \partial_{\mu} G(x) \partial_{0}^{*} \partial_{\nu} \partial_{\nu} G(x)=2 n_{1} \mathcal{F}_{4},
\end{aligned}
$$

where $\mathcal{F}_{4}$ is given by (B.9).

\section{C.2 Correlators appearing in $F_{2,0}$ and $F_{2,1}$}

Firstly

$$
\left\langle W_{2}\right\rangle_{0}=n_{1}\left(n_{1}-1\right)\left(I_{21}-\frac{1}{4} I_{22}\right)
$$

Next

$$
\begin{aligned}
\left\langle W_{3}\right\rangle_{0} & =\frac{n_{1}}{V} \sum_{x y} G(x-y)\left\{n_{1} G(x-y) \nabla_{0}^{x} \nabla_{0}^{y} G(x-y)+2\left[\nabla_{0}^{x} G(x-y)\right]\left[\nabla_{0}^{y} G(x-y)\right]\right\} \\
& =-n_{1}^{2} \sum_{x} G(x)^{2} \nabla_{0}^{2} G(x)-2 n_{1} \sum_{x}\left[\nabla_{0} G(x)\right]^{2} G(x) \\
& =n_{1}\left(n_{1}-1\right) W_{3 a}+\frac{1}{2} n_{1} W_{3 c}
\end{aligned}
$$

where $W_{3 a}, W_{3 c}$ are defined in (2.83), (2.84) respectively. For the connected correlators we get

$$
\begin{aligned}
\left\langle W_{2} A_{2,0}\right\rangle_{0}^{c}= & n_{1} \sum_{x y \mu}\left\{n_{1} G(x-y) \partial_{\mu}^{*} \nabla_{0} G(x-z) \partial_{\mu}^{*} \nabla_{0} G(y-z)\right. \\
& -n_{1} \nabla_{0} \nabla_{0} G(x-y) \partial_{\mu}^{*} G(x-z) \partial_{\mu}^{*} G(y-z) \\
& \left.+2 \nabla_{0} G(x-y) \partial_{\mu}^{*} \nabla_{0} G(x-z) \partial_{\mu}^{*} G(y-z)\right\} \\
= & 2 n_{1}\left(n_{1}-1\right)\left[I_{21}-\frac{1}{4} I_{22}\right] \\
\left\langle W_{2} A_{2,1}^{(a)}\right\rangle_{0}^{c}= & -2 n_{1}\left(n_{1}-1\right)\left(1-\frac{n_{1}}{V}\right) \frac{1}{V} \sum_{x y u} G(x-u) G(y-u) \nabla_{0}^{x} \nabla_{0}^{y} G(x-y) \\
= & -2 n_{1}\left(n_{1}-1\right)\left(1-\frac{n_{1}}{V}\right) \frac{1}{V} \sum_{p}^{\prime} \frac{\sin ^{2} p_{0}}{\left(\hat{p}^{2}\right)^{3}} \\
= & -2 n_{1}\left(n_{1}-1\right)\left(1-\frac{n_{1}}{V}\right)\left(I_{31}-\frac{1}{4} I_{32}\right)
\end{aligned}
$$

\footnotetext{
${ }^{17}$ Note that one can obtain the results of insertions in eqs. (C.3) and (C.6)-(C.10) by observing that $\left\langle X A_{2,0}\right\rangle_{0}^{c}$ inserts for each propagator appearing in $\langle X\rangle_{0}$ a factor 1, i.e. simply counts the number of propagators in $\langle X\rangle_{0}$. Similarly, for the other operators the corresponding insertions are $A_{4,1}^{(1)} \rightarrow 2 \hat{p}^{2}$, $A_{4,1}^{(5 a)} \rightarrow 2 \sum_{\mu} \hat{p}_{\mu}^{4} / \hat{p}^{2}$, and $A_{4,1}^{(5 c)} \rightarrow 2 \sum_{\mu \nu} \cos \left(p_{\mu}-p_{\nu}\right) \hat{p}_{\mu}^{2} \hat{p}_{\nu}^{2} / \hat{p}^{2}$.
} 


$$
\begin{aligned}
\left\langle W_{2} A_{2,1}^{(b)}\right\rangle_{0}^{c} & =\left.\frac{1}{8 V} \sum_{x y u} \partial_{\mu}^{u} \partial_{\mu}^{v}\left\langle\left[\nabla_{0} \vec{\pi}(x) \cdot \nabla_{0} \vec{\pi}(y)\right] \vec{\pi}(x) \cdot \vec{\pi}(y) \vec{\pi}(u)^{2} \vec{\pi}(v)^{2}\right\rangle_{0}^{c}\right|_{v=u} \\
& =n_{1}\left(n_{1}-1\right) \frac{1}{V} \sum_{x y u} \partial_{\mu}^{u} \partial_{\mu}^{v}[G(x-u) G(y-v) \times \\
\{2 G(u-v) & \left.\left.\nabla_{0}^{x} \nabla_{0}^{y} G(x-y)-\nabla_{0}^{x} G(x-v) \nabla_{0}^{y} G(y-u)\right\}\right]_{v=u} \\
= & n_{1}\left(n_{1}-1\right)\left[2 \mathcal{F}_{2}-\mathcal{F}_{3}\right],
\end{aligned}
$$

with $\mathcal{F}_{2}, \mathcal{F}_{3}$ defined in (B.7), (B.8).

$$
\begin{aligned}
\left\langle W_{2} A_{4,1}^{(1)}\right\rangle_{0}^{c} & =4 n_{1}\left(n_{1}-1\right)\left[I_{11}-\frac{1}{4} I_{12}\right], \\
\left\langle W_{2} A_{4,1}^{(i)}\right\rangle_{0}^{c} & =0, \quad i=2,3,4 \\
\left\langle W_{2} A_{4,1}^{(5 a)}\right\rangle_{0}^{c} & =4 n_{1}\left(n_{1}-1\right)\left[J_{31}-\frac{1}{4} J_{32}\right], \\
\left\langle W_{2} A_{4,1}^{(5 b)}\right\rangle_{0}^{c} & =4 n_{1}\left(n_{1}-1\right)\left[I_{11}-\frac{1}{4} I_{12}\right] \\
\left\langle W_{2} A_{4,1}^{(5 c)}\right\rangle_{0}^{c} & =4 n_{1}\left(n_{1}-1\right) \mathcal{F}_{5}
\end{aligned}
$$

where $\mathcal{F}_{5}$ is given by (B.10).

\section{C.3 Computation of $F_{3}$ up to $\mathrm{O}\left(g_{0}^{2}\right)$}

We have

$$
F_{3}=\sum_{i=1}^{5} \frac{g_{4}^{(i)}}{4} F_{3}^{(i)}, \quad F_{3}^{(i)}=\frac{1}{V}\left\langle C_{4}^{(i)}\right\rangle_{\mathcal{A}} .
$$

Averaging over the rotations gives the following expressions for the $F_{3}^{(i)}$ :

$$
\begin{aligned}
F_{3}^{(1)} & =F_{3}^{(5 a)}+F_{3}^{(1 a)}, \\
F_{3}^{(1 a)} & =\frac{2}{n} \frac{1}{V} \sum_{x k}\left\langle\left[2 \mathbf{S}(x)+\square_{0} \mathbf{S}(x)\right] \cdot \square_{k} \mathbf{S}(x)\right\rangle_{\mathcal{A}} . \\
F_{3}^{(2)} & =-\frac{4}{n n_{1}} \frac{1}{V} \sum_{x}\left\langle 2-2\left\{\mathbf{S}(x) \cdot \mathbf{S}^{\prime}(x)\right\}^{2}+n_{1}\left\{\mathbf{S}(x) \cdot \mathbf{S}^{\prime}(x)\right\} \partial_{\mu} \mathbf{S}(x) \cdot \partial_{\mu} \mathbf{S}(x)\right\rangle_{\mathcal{A}},
\end{aligned}
$$

where we have introduced the notation $S_{i}^{\prime}(x)=S_{i}(x+\hat{0})$ and below $S_{i}^{\prime \prime}(x)=S_{i}(x+2 \hat{0})$.

$$
\begin{aligned}
F_{3}^{(3)}= & F_{3}^{(4 a)}+F_{3}^{(3 a)}, \\
F_{3}^{(3 a)}= & \frac{4}{n n_{1}} \frac{1}{V} \sum_{x k}\left\langle\mathbf{S}^{\prime}(x) \cdot \partial_{k} \mathbf{S}(x)\left[n_{1} \partial_{0} \mathbf{S}(x) \cdot \partial_{k} \mathbf{S}(x)+\mathbf{S}^{\prime}(x) \cdot \partial_{k} \mathbf{S}(x)\right]\right. \\
& \left.-\partial_{k} \mathbf{S}(x) \cdot \partial_{k} \mathbf{S}(x)\right\rangle_{\mathcal{A}} \cdot \\
F_{3}^{(4)}= & F_{3}^{(4 a)}-\frac{1}{d+2}\left(F_{3}^{(2)}+2 F_{3}^{(3)}\right),
\end{aligned}
$$

with

$$
F_{3}^{(4 a)}=-\frac{4}{n n_{1}} \frac{1}{V} \sum_{x}\left\langle\left\{n_{1}\left(\mathbf{S}(x) \cdot \mathbf{S}^{\prime}(x)\right) \partial_{0} \mathbf{S}(x) \cdot \partial_{0} \mathbf{S}(x)+2-2\left(\mathbf{S}(x) \cdot \mathbf{S}^{\prime}(x)\right)^{2}\right\}\right\rangle_{\mathcal{A}} .
$$


Next

$$
\begin{aligned}
F_{3}^{(5 a)} & =-\frac{4}{n} \frac{1}{V} \sum_{x}\left\langle-\mathbf{S}(x) \cdot \square_{0} \mathbf{S}(x)+2 \partial_{0} \mathbf{S}(x) \cdot \partial_{0}^{*} \mathbf{S}(x)\right\rangle_{\mathcal{A}} . \\
F_{3}^{(5 b)} & =F_{3}^{(1)} \\
F_{3}^{(5 c)} & =F_{3}^{(5 a)}+F_{3}^{(5 d)} \\
F_{3}^{(5 d)} & =\frac{4}{n} \frac{1}{V} \sum_{x k}\left\langle\left[2 \mathbf{S}^{\prime \prime}(x)-\mathbf{S}^{\prime}(x)\right] \cdot \partial_{k}^{2} \mathbf{S}(x)\right\rangle_{\mathcal{A}} .
\end{aligned}
$$

$F_{3}$ has a perturbative expansion starting at $\mathrm{O}\left(g_{0}^{2}\right)$ :

$$
F_{3}=\sum_{r=1} F_{3, r} g_{0}^{2 r}, \quad F_{3}^{(i)}=\sum_{r=1} F_{3, r}^{(i)} g_{0}^{2 r} .
$$

\section{C.3.1 Computation of $F_{3,1}^{(i)}$}

$$
\begin{aligned}
F_{3,1}^{(1)} & =F_{3,1}^{(5 a)}+F_{3,1}^{(1 a)}, \\
F_{3,1}^{(1 a)} & =\frac{2}{n} \frac{1}{V} \sum_{x k}\left\langle\left[2 \vec{\pi}(x)+\square_{0} \vec{\pi}(x)\right] \cdot \square_{k} \vec{\pi}(x)\right\rangle_{0} \\
& =\frac{2 n_{1}}{n} \sum_{k}\left[2+\square_{0}\right] \square_{k} G(0) \\
& =\frac{2 n_{1}}{n}\left[-2 I_{00}+2 I_{11}+I_{01}-I_{12}\right] . \\
F_{3,1}^{(2)} & =-\frac{4}{n n_{1}} \frac{1}{V} \sum_{x}\left\langle 2 \partial_{0} \vec{\pi}(x) \cdot \partial_{0} \vec{\pi}(x)+n_{1} \partial_{\mu} \vec{\pi}(x) \cdot \partial_{\mu} \vec{\pi}(x)\right\rangle_{0} \\
& =\frac{4}{n}\left\{2 \square_{0} G(0)+n_{1} \square G(0)\right\} \\
& =-\frac{4}{n}\left\{2 I_{11}+n_{1} I_{00}\right\} . \\
F_{3,1}^{(3)} & =F_{3,1}^{(4 a)}+F_{3,1}^{(3 a)}, \\
F_{3,1}^{(3 a)} & =-\frac{4}{n n_{1}} \frac{1}{V} \sum_{x k}\left\langle\partial_{k} \vec{\pi}(x) \cdot \partial_{k} \vec{\pi}(x)\right\rangle_{0} \\
& =-\frac{4}{n}\left[I_{00}-I_{11}\right] . \\
F_{3,1}^{(4 a)} & =-\frac{4\left(n_{1}+2\right)}{n n_{1}} \frac{1}{V} \sum_{x}\left\langle\partial_{0} \vec{\pi}(x) \cdot \partial_{0} \vec{\pi}(x)\right\rangle_{0} \\
& =-\frac{4\left(n_{1}+2\right)}{n} I_{11} \cdot \\
F_{3,1}^{(5 a)} & =-\frac{4}{n} \frac{1}{V} \sum_{x}\left\langle-\vec{\pi}(x) \cdot \square \square_{0} \vec{\pi}(x)+2 \partial_{0} \vec{\pi}(x) \cdot \partial_{0}^{*} \vec{\pi}(x)\right\rangle_{0} \\
& =\frac{4 n_{1}}{n}\left\{\square_{0} G(0)+2 \partial_{0}^{2} G(0)\right\} \\
& \left\{3 I_{11}-I_{12}\right\} . \\
&
\end{aligned}
$$


Finally

$$
\begin{aligned}
F_{3,1}^{(5 c)} & =F_{3,1}^{(5 a)}+F_{3,1}^{(5 d)}, \\
F_{3,1}^{(5 d)} & =\frac{4}{n} \frac{1}{V} \sum_{x k}\left\langle\left[2 \vec{\pi}^{\prime \prime}(x)-\vec{\pi}^{\prime}(x)\right] \cdot \partial_{k}^{2} \vec{\pi}(x)\right\rangle_{0} \\
& =-\frac{4 n_{1}}{n} \mathcal{F}_{6},
\end{aligned}
$$

where $\mathcal{F}_{6}$ is given by (B.11).

\section{C.4 Computation of $F_{4}$ up to $\mathrm{O}\left(g_{0}^{2}\right)$}

$F_{4}$ is given by

$$
F_{4}=Z_{4} \sum_{i} \frac{g_{4}^{(i)}}{4} F_{4}^{(i)}, \quad F_{4}^{(i)}=\frac{1}{V}\left\langle B B_{4}^{(i)}\right\rangle_{\mathcal{A}} .
$$

Again averaging over rotations:

$$
\begin{aligned}
F_{4}^{(1)}= & F_{4}^{(5 a)}+F_{4}^{(1 a)} \\
F_{4}^{(1 a)}= & -\frac{8}{n n_{1} g_{0}^{2}} \frac{1}{V} \sum_{x y k}\left\langle\left[\mathbf{S}(x) \cdot \square_{k} \mathbf{S}(y)\right] \mathbf{S}^{\prime}(x) \cdot \nabla_{0} \mathbf{S}(y)\right. \\
& \left.-\left[\mathbf{S}(x) \cdot \nabla_{0} \mathbf{S}(y)\right] \mathbf{S}^{\prime}(x) \cdot \square_{k} \mathbf{S}(y)\right\rangle_{\mathcal{A}} \cdot \\
F_{4}^{(2)}= & \frac{8}{n n_{1} g_{0}^{2}} \frac{1}{V} \sum_{x y}\left\langle\left[\{\mathbf{S}(x) \cdot \mathbf{S}(y)\} \mathbf{S}^{\prime}(x) \cdot \mathbf{S}^{\prime}(y)\right.\right. \\
& \left.\left.-\left\{\mathbf{S}(x) \cdot \mathbf{S}^{\prime}(y)\right\} \mathbf{S}^{\prime}(x) \cdot \mathbf{S}(y)\right] \partial_{\mu} \mathbf{S}(y) \cdot \partial_{\mu} \mathbf{S}(y)\right\rangle_{\mathcal{A}} \cdot \\
F_{4}^{(3)}= & F_{4}^{(4 a)}+F_{4}^{(3 a)} \\
F_{4}^{(3 a)}= & \frac{8}{n n_{1} g_{0}^{2}} \frac{1}{V} \sum_{x y k}\left\langle\left[\left\{\mathbf{S}(x) \cdot \mathbf{S}{ }^{\prime}(y)\right\} \mathbf{S}^{\prime}(x) \cdot \partial_{k} \mathbf{S}(y)\right.\right. \\
& \left.\left.-\left\{\mathbf{S}^{\prime}(x) \cdot \mathbf{S}^{\prime}(y)\right\} \mathbf{S}(x) \cdot \partial_{k} \mathbf{S}(y)\right] \partial_{0} \mathbf{S}(y) \cdot \partial_{k} \mathbf{S}(y)\right\rangle_{\mathcal{A}} \\
F_{4}^{(4 a)}= & \frac{8}{n n_{1} g_{0}^{2}} \frac{1}{V} \sum_{x y}\left\langle\left[\{\mathbf{S}(x) \cdot \mathbf{S}(y)\} \mathbf{S}^{\prime}(x) \cdot \mathbf{S}^{\prime}(y)\right.\right. \\
& \left.\left.-\left\{\mathbf{S}(x) \cdot \mathbf{S}^{\prime}(y)\right\} \mathbf{S}^{\prime}(x) \cdot \mathbf{S}(y)\right] \partial_{0} \mathbf{S}(y) \cdot \partial_{0} \mathbf{S}(y)\right\rangle_{\mathcal{A}} . \\
F_{4}^{(5 a)}= & \frac{8}{n n_{1} g_{0}^{2}} \frac{1}{V} \sum_{x y}\left\langle\left\{\mathbf{S}(x) \cdot \partial_{0}^{*} \mathbf{S}(y)\right\} \mathbf{S}^{\prime}(x) \cdot \partial_{0} \mathbf{S}(y)-\left\{\mathbf{S}(x) \cdot \partial_{0} \mathbf{S}(y)\right\} \mathbf{S}^{\prime}(x) \cdot \partial_{0}^{*} \mathbf{S}(y)\right\rangle_{\mathcal{A}} \cdot \\
F_{4}^{(5 b)}= & F_{4}^{(1)} \cdot
\end{aligned}
$$

Finally

$$
\begin{aligned}
F_{4}^{(5 c)}= & F_{4}^{(5 a)}+F_{4}^{(5 d)} \\
F_{4}^{(5 d)}= & \frac{8}{n n_{1} g_{0}^{2}} \frac{1}{V} \sum_{x y k}\left\langle\left\{\mathbf{S}(x) \cdot\left[\mathbf{S}^{\prime \prime}(y)-\mathbf{S}^{\prime}(y)\right]\right\} \mathbf{S}^{\prime}(x) \cdot \partial_{k}^{2} \mathbf{S}(y)\right. \\
& \left.-\left\{\mathbf{S}^{\prime}(x) \cdot\left[\mathbf{S}^{\prime \prime}(y)-\mathbf{S}^{\prime}(y)\right]\right\} \mathbf{S}(x) \cdot \partial_{k}^{2} \mathbf{S}(y)\right\rangle_{\mathcal{A}} .
\end{aligned}
$$


$F_{4}$ has a perturbative expansion starting at $\mathrm{O}\left(g_{0}^{2}\right)$ :

$$
F_{4}=\sum_{r=1} F_{4, r} g_{0}^{2 r}, \quad F_{4}^{(i)}=\sum_{r=1} F_{4, r}^{(i)} g_{0}^{2 r} .
$$

\section{C.4.1 Computation of $F_{4,1}^{(i)}$}

$$
\begin{aligned}
F_{4,1}^{(1)}= & F_{4,1}^{(5 a)}+F_{4,1}^{(1 a)} \\
F_{4,1}^{(1 a)}= & -\frac{4}{n n_{1}} \frac{1}{V} \sum_{x y k}\left\langle\square_{k}^{y}\left[(\vec{\pi}(x)-\vec{\pi}(y))^{2}\right] \nabla_{0}^{y}\left[\left(\vec{\pi}^{\prime}(x)-\vec{\pi}(y)\right)^{2}\right]\right\rangle_{0} \\
= & -\frac{8}{n} \frac{1}{V} \sum_{x y k}\left[2 n_{1} \square_{k}^{y} G(x-y) \nabla_{0}^{y} G(x+\hat{0}-y)\right. \\
& \left.+\left(\square_{k}^{y} \nabla_{0}^{z}\{G(\hat{0})-G(x-z)-G(y-x-\hat{0})+G(y-z)\}^{2}\right)_{z=y}\right] \\
= & \frac{16\left(n_{1}-1\right)}{n}\left\{I_{11}-\frac{1}{4} I_{12}-I_{22}+\frac{1}{4} I_{23}\right\} \cdot \\
F_{4,1}^{(2)}= & \frac{8}{n n_{1}} \frac{1}{V} \sum_{x y}\left\langle\left\{\partial_{0} \vec{\pi}(x) \cdot \partial_{0} \vec{\pi}(y)\right\} \partial_{\mu} \vec{\pi}(y) \cdot \partial_{\mu} \vec{\pi}(y)\right\rangle_{0}=0 . \\
F_{4,1}^{(3)}= & F_{4,1}^{(4 a)}+F_{4,1}^{(3 a)}, \\
F_{4,1}^{(3 a)}= & \frac{8}{n n_{1}} \frac{1}{V} \sum_{x y k}\left\langle\left\{\partial_{0} \vec{\pi}(x) \cdot \partial_{k} \vec{\pi}(y)\right\} \partial_{0} \vec{\pi}(y) \cdot \partial_{k} \vec{\pi}(y)\right\rangle_{0}=0 . \\
F_{4,1}^{(4 a)}= & \frac{8}{n n_{1}} \frac{1}{V} \sum_{x y}\left\langle\left\{\partial_{0} \vec{\pi}(x) \cdot \partial_{0} \vec{\pi}(y)\right\} \partial_{0} \vec{\pi}(y) \cdot \partial_{0} \vec{\pi}(y)\right\rangle_{0}=0 . \\
F_{4,1}^{(5 a)}= & \frac{8}{n n_{1}} \frac{1}{V} \sum_{x y}\left\langle\left\{\vec{\pi}(x) \cdot \partial_{0}^{*} \vec{\pi}(y)\right\} \vec{\pi}^{\prime}(x) \cdot \partial_{0} \vec{\pi}(y)-\left\{\vec{\pi}(x) \cdot \partial_{0} \vec{\pi}(y)\right\} \vec{\pi}^{\prime}(x) \cdot \partial_{0}^{*} \vec{\pi}(y)\right\rangle_{0} \\
= & \frac{8\left(n_{1}-1\right)}{n} \frac{1}{V} \sum_{x y}\left\{\partial_{0}^{* y} G(x-y) \partial_{0}^{y} G(x+\hat{0}-y)-\partial_{0}^{y} G(x-y) \partial_{0}^{* y} G(x+\hat{0}-y)\right\} \\
= & \frac{16\left(n_{1}-1\right)}{n}\left\{I_{22}-\frac{1}{4} I_{23}\right\}
\end{aligned}
$$

and finally

$$
\begin{aligned}
F_{4,1}^{(5 c)}= & F_{4,1}^{(5 a)}+F_{4,1}^{(5 d)} \\
F_{4,1}^{(5 d)=} & \frac{8}{n n_{1}} \frac{1}{V} \sum_{x y k}\left\langle\left\{\vec{\pi}(x) \cdot\left[\vec{\pi}^{\prime \prime}(y)-\vec{\pi}^{\prime}(y)\right]\right\} \vec{\pi}^{\prime}(x) \cdot \partial_{k}^{2} \vec{\pi}(y)\right. \\
& \left.-\left\{\vec{\pi}^{\prime}(x) \cdot\left[\vec{\pi}^{\prime \prime}(y)-\vec{\pi}^{\prime}(y)\right]\right\} \vec{\pi}(x) \cdot \partial_{k}^{2} \vec{\pi}(y)\right\rangle_{0} \\
= & \frac{8\left(n_{1}-1\right)}{n} \frac{1}{V} \sum_{x y k}\left[\partial_{0}^{y} G(x-y-\hat{0}) \partial_{k}^{y 2} G(x+\hat{0}-y)-\partial_{0}^{y} G(x-y) \partial_{k}^{y 2} G(x-y)\right] \\
= & \frac{8\left(n_{1}-1\right)}{n} \mathcal{F}_{7}
\end{aligned}
$$

where $\mathcal{F}_{7}$ is given by (B.12). 


\section{C.5 Computation of $F_{5}$ up to $\mathrm{O}\left(g_{0}^{2}\right)$}

$$
F_{5}=\mathrm{O}\left(g_{0}^{4}\right),
$$

and hence doesn't contribute to the order considered.

\section{The $\mathbf{n}=2$ case with lattice regularization}

The lattice action with the chemical potential is

$$
\begin{aligned}
A & =-\frac{1}{g_{0}^{2}} \sum_{x \mu} \cos \left(\partial_{\mu} \Phi(x)-i h \delta_{\mu 0}\right) \\
& =-\frac{1}{g_{0}^{2}} \sum_{x}\left[\sum_{\mu} \cos \left(\partial_{\mu} \Phi(x)\right)+i h \sin \left(\partial_{0} \Phi(x)\right)+\frac{1}{2} h^{2} \cos \left(\partial_{0} \Phi(x)\right)\right]+\mathrm{O}\left(h^{3}\right)
\end{aligned}
$$

With $\Phi(x)=g_{0} \phi(x)$ we have

$$
\left.A\right|_{h=0}=A_{0}+g_{0}^{2} A_{1}+g_{0}^{4} A_{2}+\ldots
$$

where

$$
\begin{aligned}
& A_{0}=\frac{1}{2} \sum_{x \mu}\left(\partial_{\mu} \phi(x)\right)^{2}, \\
& A_{1}=-\frac{1}{24} \sum_{x \mu}\left(\partial_{\mu} \phi(x)\right)^{4} .
\end{aligned}
$$

The $h$ dependent part is given by

$$
\begin{aligned}
A_{h} & =-\frac{h^{2}}{2 g_{0}^{2}} V+i h g_{0} B_{1}+h^{2}\left(B_{20}+g_{0}^{2} B_{21}+\ldots\right) \\
B_{1} & =\frac{1}{6} \sum_{x}\left(\partial_{0} \phi(x)\right)^{3}, \\
B_{20} & =\frac{1}{4} \sum_{x}\left(\partial_{0} \phi(x)\right)^{2}, \\
B_{21} & =-\frac{1}{48} \sum_{x}\left(\partial_{0} \phi(x)\right)^{4} .
\end{aligned}
$$

Note that we need the free energy only up to $h^{2} g_{0}^{2}$; the omitted terms do not contribute to this order.

$$
\begin{aligned}
V f_{h} & =\left\langle A_{h}\right\rangle-\frac{1}{2}\left\langle A_{h}^{2}\right\rangle+\frac{1}{2}\left\langle A_{h}\right\rangle^{2} \ldots \\
& =-\frac{h^{2}}{2 g_{0}^{2}} V+h^{2}\left\langle B_{20}\right\rangle_{0}+h^{2} g_{0}^{2}\left(\left\langle B_{21}\right\rangle_{0}-\left\langle B_{20} A_{1}\right\rangle_{0}^{c}+\frac{1}{2}\left\langle B_{1}^{2}\right\rangle_{0}\right) .
\end{aligned}
$$

So

$$
\chi=\frac{1}{g_{0}^{2}}\left(1+g_{0}^{2} R_{1}+g_{0}^{4} R_{2}+\ldots\right),
$$


with

$$
\begin{aligned}
R_{1} & =-\frac{2}{V}\left\langle B_{20}\right\rangle_{0}=-\frac{1}{2}\left\langle\frac{1}{V} \sum_{x}\left(\partial_{0} \phi(x)\right)^{2}\right\rangle_{0} \\
& =\frac{1}{2} \square_{0} G(0)=-\frac{1}{2 V} \sum_{p}^{\prime} \frac{\hat{p}_{0}^{2}}{\hat{p}^{2}}=-\frac{1}{2} I_{11},
\end{aligned}
$$

in agreement with (3.55), and

$$
\begin{aligned}
R_{2}= & -\frac{2}{V}\left\langle B_{21}\right\rangle_{0}+\frac{2}{V}\left\langle B_{20} A_{1}\right\rangle_{0}^{c}-\frac{1}{V}\left\langle B_{1}^{2}\right\rangle_{0} \\
= & \frac{1}{24}\left\langle\frac{1}{V} \sum_{x}\left(\partial_{0} \phi(x)\right)^{4}\right\rangle_{0}-\frac{1}{48}\left\langle\frac{1}{V} \sum_{x y \mu}\left(\partial_{0} \phi(x)\right)^{2}\left(\partial_{\mu} \phi(y)\right)^{4}\right\rangle_{0}^{c} \\
& -\frac{1}{36}\left\langle\frac{1}{V} \sum_{x y}\left(\partial_{0} \phi(x)\right)^{3}\left(\partial_{0} \phi(y)\right)^{3}\right\rangle_{0} \\
= & \frac{1}{8}\left(\square_{0} G(0)\right)^{2}+\frac{1}{4} \sum_{x \mu} \square_{0} G(x) \square_{\mu} G(x) \square_{\mu} G(0)+\frac{1}{6} \sum_{x}\left(\square_{0} G(x)\right)^{3} \\
= & \frac{1}{8}\left(\frac{1}{V} \sum_{p}^{\prime} \frac{\hat{p}_{0}^{2}}{\hat{p}^{2}}\right)^{2}-\frac{1}{4} \sum_{\mu}\left[\left(\frac{1}{V} \sum_{p}^{\prime} \frac{\hat{p}_{0}^{2} \hat{p}_{\mu}^{2}}{\left(\hat{p}^{2}\right)^{2}}\right)\left(\frac{1}{V} \sum_{q}^{\prime} \frac{\hat{q}_{\mu}^{2}}{\hat{q}^{2}}\right)\right]-\frac{1}{6} S_{n 2} \\
= & \frac{1}{8} I_{11}^{2}-\frac{1}{4} I_{22} I_{11}-\frac{1}{4 d_{s}}\left(I_{11}-I_{22}\right)\left(I_{00}-I_{11}\right)-W_{3 c} .
\end{aligned}
$$

Here

$$
S_{n 2}=-\sum_{x}\left(\square_{0} G(x)\right)^{3}=6 W_{3 c} .
$$

The last equation follows from the direct comparison with (2.84). Also

$$
-\sum_{x \mu} \square_{0} G(x) \square_{\mu} G(x) \square_{\mu} G(0)=I_{22} I_{11}+\frac{1}{d_{s}}\left(I_{11}-I_{22}\right)\left(I_{00}-I_{11}\right) .
$$

The result for $R_{2}$ above agrees with the result in (2.88) for $n_{1}=1$.

Open Access. This article is distributed under the terms of the Creative Commons Attribution License (CC-BY 4.0), which permits any use, distribution and reproduction in any medium, provided the original author(s) and source are credited.

\section{References}

[1] S. Weinberg, Phenomenological Lagrangians, Physica A 96 (1979) 327 [InSPIRE].

[2] J. Gasser and H. Leutwyler, Chiral Perturbation Theory to One Loop, Annals Phys. 158 (1984) 142 [inSPIRE].

[3] S. Aoki et al., Review of lattice results concerning low-energy particle physics, Eur. Phys. J. C 74 (2014) 2890 [arXiv:1310.8555] [InSPIRE].

[4] H. Leutwyler, Energy Levels of Light Quarks Confined to a Box, Phys. Lett. B 189 (1987) 197 [INSPIRE]. 
[5] P. Hasenfratz, The QCD rotator in the chiral limit, Nucl. Phys. B $\mathbf{8 2 8}$ (2010) 201 [arXiv:0909.3419] [INSPIRE].

[6] P. Hasenfratz and F. Niedermayer, Finite size and temperature effects in the AF Heisenberg model, Z. Phys. B 92 (1993) 91 [hep-lat/9212022] [INSPIRE].

[7] F. Niedermayer and C. Weiermann, The rotator spectrum in the delta-regime of the $O(n)$ effective field theory in 3 and 4 dimensions, Nucl. Phys. B 842 (2011) 248 [arXiv: 1006.5855] [INSPIRE].

[8] M. Lüscher, P. Weisz and U. Wolff, A Numerical method to compute the running coupling in asymptotically free theories, Nucl. Phys. B 359 (1991) 221 [INSPIRE].

[9] F. Niedermayer and P. Weisz, Massless sunset diagrams in finite asymmetric volumes, arXiv: 1602.03159 [INSPIRE].

[10] M. Weingart, The QCD rotator with a light quark mass, arXiv:1006.5076 [INSPIRE].

[11] M. Weingart, QCD rotator with light quarks up to NNL order, PoS (Lattice 2010) 094.

[12] M.E. Matzelle and B.C. Tiburzi, Low-Energy QCD in the Delta Regime, Phys. Rev. D 93 (2016) 034506 [arXiv: 1512.05286] [INSPIRE].

[13] P. Hasenfratz, Perturbation theory and zero modes in $O(N)$ lattice $\sigma$-models, Phys. Lett. B 141 (1984) 385 [INSPIRE].

[14] E. Brézin, J. Zinn-Justin and J.C. Le Guillou, Renormalization of the nonlinear $\sigma$-model in $2+\epsilon$ dimension, Phys. Rev. D 14 (1976) 2615 [inSPIRE].

[15] D.J. Amit and G.B. Kotliar, Nonlinear $\sigma$-model and $C P^{(n-1)}$ at $(2+\epsilon)$-dimensions, Nucl. Phys. B 170 (1980) 187 [INSPIRE].

[16] P. Hasenfratz and H. Leutwyler, Goldstone Boson Related Finite Size Effects in Field Theory and Critical Phenomena With O(N) Symmetry, Nucl. Phys. B 343 (1990) 241 [InSPIRE].

[17] P. Hasenfratz, M. Maggiore and F. Niedermayer, The Exact mass gap of the $O(3)$ and $O(4)$ nonlinear $\sigma$-models in $D=2$, Phys. Lett. B 245 (1990) 522 [INSPIRE].

[18] P. Hasenfratz and F. Niedermayer, The Exact mass gap of the $O(N) \sigma$-model for arbitrary $n \geq 3$ in $D=2$, Phys. Lett. B 245 (1990) 529 [INSPIRE].

[19] J. Balog and A. Hegedüs, TBA Equations for excited states in the $O(3)$ and $O(4)$ nonlinear б-model, J. Phys. A 37 (2004) 1881 [hep-th/0309009] [INSPIRE].

[20] A. Hegedüs, Nonlinear integral equations for finite volume excited state energies of the $O(3)$ and $O(4)$ nonlinear $\sigma$-models, J. Phys. A 38 (2005) 5345 [hep-th/0412125] [InSPIRE].

[21] J. Balog and A. Hegedüs, TBA equations for the mass gap in the $O(2 r)$ non-linear $\sigma$-models, Nucl. Phys. B 725 (2005) 531 [hep-th/0504186] [INSPIRE].

[22] J. Balog and A. Hegedüs, The finite size spectrum of the 2-dimensional $O(3)$ nonlinear б-model, Nucl. Phys. B 829 (2010) 425 [arXiv:0907.1759] [INSPIRE].

[23] G. Parisi, On the Relation Between the Renormalized and the Bare Coupling Constant on the Lattice, Phys. Lett. B 92 (1980) 133 [inSPIRE].

[24] M. Falcioni and A. Treves, The nonlinear $\sigma$ model: 3 loops renormalization and lattice scaling, Nucl. Phys. B 265 (1986) 671 [InSPIRE].

[25] QCDSF collaboration, W. Bietenholz et al., QCD in the delta-Regime, J. Phys. Conf. Ser. 287 (2011) 012016 [arXiv:1103.3311] [INSPIRE]. 
[26] W. Bietenholz et al., Pion in a Box, Phys. Lett. B 687 (2010) 410 [arXiv:1002.1696] [INSPIRE].

[27] W. Bietenholz, Chiral fermions on the lattice, AIP Conf. Proc. 1361 (2011) 245 [arXiv: 1007.0285] [INSPIRE].

[28] G. Colangelo, J. Gasser and H. Leutwyler, $\pi \pi$ scattering, Nucl. Phys. B 603 (2001) 125 [hep-ph/0103088] [INSPIRE].

[29] G. Colangelo and S. Dürr, The pion mass in finite volume, Eur. Phys. J. C 33 (2004) 543 [hep-lat/0311023] [INSPIRE].

[30] M. Lüscher, Exact chiral symmetry on the lattice and the Ginsparg-Wilson relation, Phys. Lett. B 428 (1998) 342 [hep-lat/9802011] [INSPIRE].

[31] Y. Kikukawa and A. Yamada, Axial vector current of exact chiral symmetry on the lattice, Nucl. Phys. B 547 (1999) 413 [hep-lat/9808026] [INSPIRE].

[32] P. Hasenfratz, S. Hauswirth, T. Jorg, F. Niedermayer and K. Holland, Testing the fixed point QCD action and the construction of chiral currents, Nucl. Phys. B 643 (2002) 280 [hep-lat/0205010] [INSPIRE]. 